\title{
OTEC Thermal Resource Report for Mombasa
}

May 1979

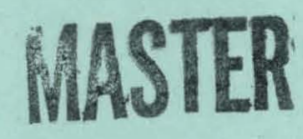

Prepared for U.S. Department of Energy Assistant Secretary for Energy Technology Division of Central Solar Technology

Under Contract No. ET-78-C-01-2898 


\section{DISCLAIMER}

This report was prepared as an account of work sponsored by an agency of the United States Government. Neither the United States Government nor any agency Thereof, nor any of their employees, makes any warranty, express or implied, or assumes any legal liability or responsibility for the accuracy, completeness, or usefulness of any information, apparatus, product, or process disclosed, or represents that its use would not infringe privately owned rights. Reference herein to any specific commercial product, process, or service by trade name, trademark, manufacturer, or otherwise does not necessarily constitute or imply its endorsement, recommendation, or favoring by the United States Government or any agency thereof. The views and opinions of authors expressed herein do not necessarily state or reflect those of the United States Government or any agency thereof. 


\section{DISCLAIMER}

Portions of this document may be illegible in electronic image products. Images are produced from the best available original document. 
Avallable from:

National Technical Information Service (NTIS)

U.S. Department of Commerce

5285 Port Royal Road

Springfield, Virginia 22161

Price: Printed copy: $\$ 4.50$

Microfiche: $\$ 3.00$ 
$\mathrm{HCP} / \mathrm{T} 2898-01 / 4$

Dist Cat UC-

\section{OTEC Thermal Resource Report for Mombasa}

May 1979

Prepared for

U.S. Department of Energy

Assistant Secretary for Energy Technology

Division of Central Solar Technology

Washington, D.C. 20585

Prepared by

William Aian Wolff

Ocean Data Systems, Inc.

Monterey, California 93940

Under Contract No. ET-78-C-01-2898

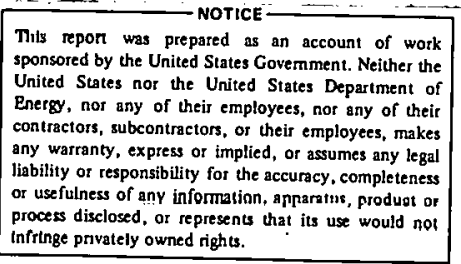




\section{NOTICE}

This report was prepared as an account of work sponsored by the United States Government. Neither the United States nor the United States Department of Energy, nor any of their employees, makes any warranty, express or implied, or assumes any legal liability or responsibility for the accuracy, completeness, or usefulness of any intormation, apparatus, product, or process disclosed, or represents that its use would not infringe privately owned rights. Reference herein to any specific commercial product, process, or service by trade name, mark, manufacturer, or otherwise, does not necessarily constitute or imply its endorsement, recommendation, or favoring by

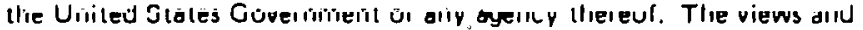
opinions of authors expressed herein do not necessarily state or reflect those of the United States Government or any agency therenfi 


\section{Section}

$\underline{\text { Page }}$

INTRODUCTION.........................

I. EXECUTIVE SUMMARY ................. I-I

II $\quad$ BATHYMETRY ................... II

III. THERMAL RESOURCE................ III

IV. MIXED LAYER DEPTH................ IV-I

v. WEATHER CONDITIONS................ v-l

VI. SEA AND SWELL CHARACTERISTICS........... VI-1

VII. CURRENTS..................... VII-I

REFERENCES.............................. R- 
- One of the basic environmental considerations in site selection for an Ocean Thermal Energy Conversion (OTEC) power plant is the availability of an adequate temperature difference resource. OTEC plants are designed to convert the potential energy in the temperature difference between the warm ocean surface water and the cold water existing at deeper depths into electricity. The turbines which produce the electricity must run on temperature differentials which are extremely small by the standards of conventional energy plants. Therefore, a definition of the most probable temperature structure fór a site is most important.

In order to define temperature structures for OTEC. areas of interest, Ocean Data systems, Inc. (ODSI) has. developed computer data files of all unclassified soundings available. The primary sources for the data were NOAA's National Oceanographic Data Center, and the U.S. Navy's Fleet Numerical Weather Central; The files were updated in September 1978. Included in the data base were mechanical bathythermographs (MBT), expendable bathythermographs (XBT), salinity temperature depth systems (STD), and Nansen casts.

Under U.S. Department of Energy, Division of Solar Energy Contract No: EY-78-C-2989, ODSI has analyzed the monthly temperature structure for ten different geographical areas. The ten sites selected for study by the Department of Energy are: 
Bottom Mounted

or

Shore Plants: Dampier Land $13-18^{\circ} \mathrm{S} \quad 118-121^{\circ} \mathrm{E}$

$\begin{array}{lcc}\text { Manila } & 14-16^{\circ} \mathrm{N} & 118-120^{\circ} \mathrm{E} \\ \text { Guam } & 12-15^{\circ} \mathrm{N} & 142-146^{\circ} \mathrm{E} \\ \text { Ivory Coast } & 3-6^{\circ} \mathrm{N} & 3-8^{\circ} \mathrm{W} \\ \text { Jakarta } & 6-9^{\circ} \mathrm{S} & 104-109^{\circ} \mathrm{E} \\ \text { Mexico } & 20-23^{\circ} \mathrm{N} & 105-110^{\circ} \mathrm{W} \\ \text { Sri Lanka } & 5-10^{\circ} \mathrm{N} & 78-83^{\circ} \mathrm{E} \\ \text { Mombasa } & 9^{\circ} \mathrm{S}-3^{\circ} \mathrm{N} & 40-45^{\circ} \mathrm{E} \\ \text { No. } 1 & 5-10^{\circ} \mathrm{N} & 90-95^{\circ} \mathrm{W} \\ \text { No. } 2 & 13-15^{\circ} \mathrm{N} & 75-80^{\circ} \mathrm{W}\end{array}$

The location of these sites is shown on the following map; a separate report was produced for each sitc.

For each area, the most probable temperature structures were determined. When the most probable temperature soundings were plotted, some month to month variability was present which was caused by the non-uniform dat.a sample rather than by real changes in the ocean. These short-period time variations were removed by a filtering process described by Wolff, et al (1977), [44]. Availability of cold and warm water was examined at each site. In addition to warm and cold water availability, there are other requirements for the continunis operation of an OTEC plant. An adequate temperature differential $(\Delta T)$ is the primary need. $A \Delta T$ greater than $16.7^{\circ} \mathrm{C}\left(30^{\circ} \mathrm{F}\right)$ for the coldest month of the year would enable year round operation. The annual mean $\Delta T$ for 


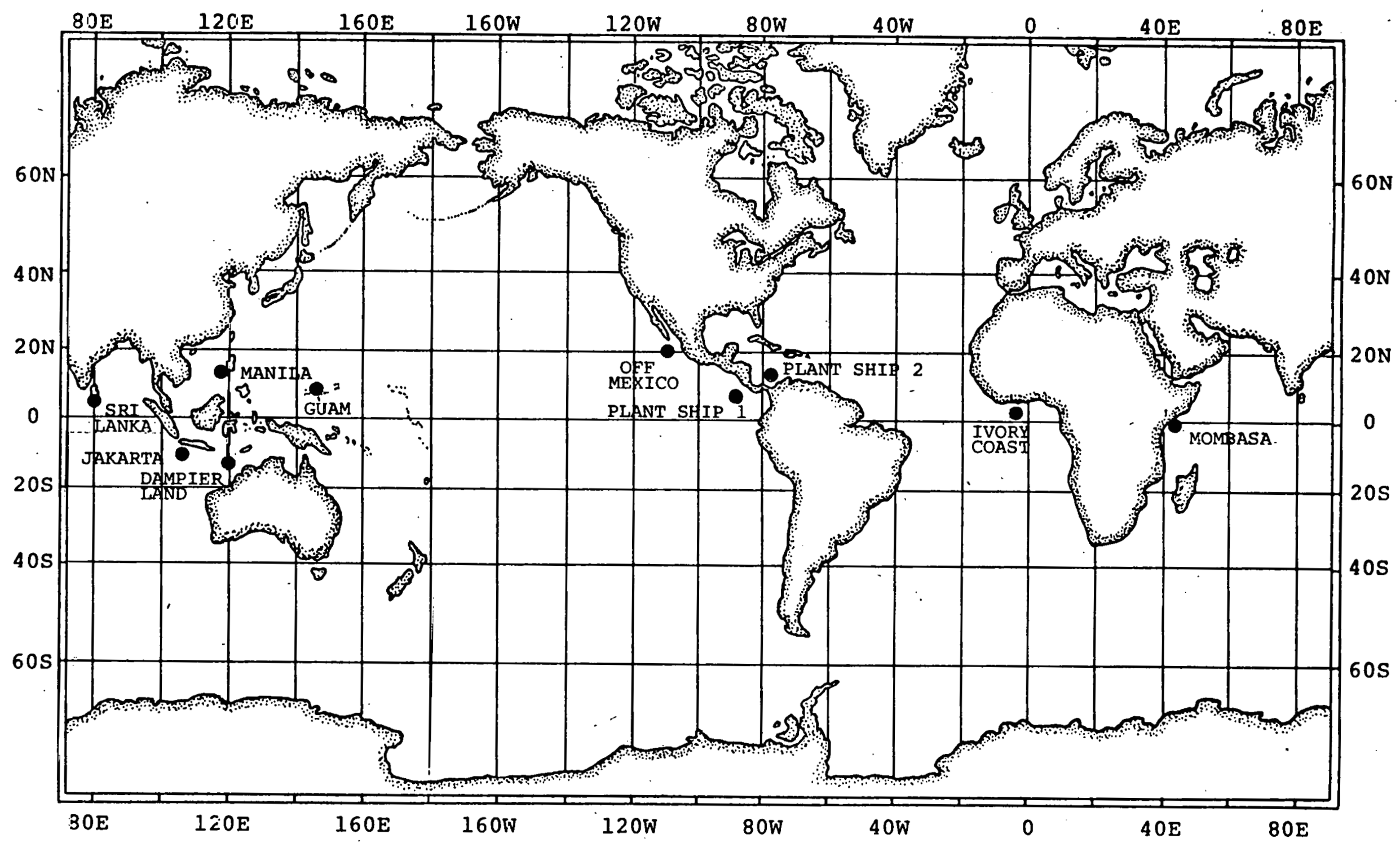

LOCATION OF THE TEN SELECTED SITES 
a site should equal or exceed $20^{\circ} \mathrm{C}\left(36^{\circ} \mathrm{F}\right)$. Besides the thermal resource, there are other operational requirements. Bottom depth should be less than 1500 meters for mooring. Minimum distances offshore to 1000-meter depth is another important consideration for shore plants. Currents should be sufficient to guarantee good cold/warm water sources and to provide for dispersion of modified water. Desirable sites also have light winds, minimum sea and swell, and the lack of severe storms. These parameters have been examined for each site. The following tables summarize some of the key site parameters. for each location. 
SUMMARY OF SITE PARAMETERS

\begin{tabular}{|c|c|c|c|c|c|c|}
\hline Site & $\begin{array}{l}\text { Monthly Mean } \\
\text { Temperature }\left({ }^{\circ} \mathrm{C}\right) \\
\text { Surface Range } \\
\end{array}$ & $\begin{array}{c}\text { Monthly Mean } \\
\text { Temperature } \cdot\left({ }^{\circ} \mathrm{C}\right) \\
1000 \mathrm{M} \\
\end{array}$ & $\begin{array}{c}\text { Annual } \\
\text { Mean } \\
\Delta \mathrm{T}\left({ }^{\circ} \mathrm{C}\right) 500 \mathrm{M} \\
\end{array}$ & 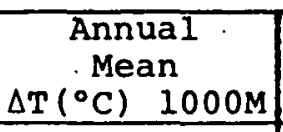 & $\begin{array}{c}\text { Coldest Monthly } \\
\text { Mean } \Delta \mathrm{T}\left({ }^{\circ} \mathrm{C}\right) \\
500 \mathrm{M} \\
\end{array}$ & $\begin{array}{c}\text { Coldest Monthly } \\
\text { Mean } \Delta \mathrm{T} \cdot\left({ }^{\circ} \mathrm{C}\right) \\
1000 \mathrm{M}\end{array}$ \\
\hline Sri Lanka & $27.5-28.6$ & $6.5-6.7$ & 18.0 & 21.3 & 17.5 & $20.8=$ \\
\hline Mombasa $\mathrm{N}$ & $25.4-28: 7$ & $7.2-7.5$ & 17.0 & 19.5 & 15.6 & 18.2 \\
\hline Mombasa $\mathbf{s}$ & $25.5-28.4$ & $6.3-7.0$ & 18.0 & 20.2 & 15.9 & 18.5 \\
\hline Jakarta & $27.2-29.0$ & $5.1-5: 6$ & 19.1 & 22.8 & 17.9 & 21.9 \\
\hline Manila & $27.1-29.5$ & $4.4-4.6$ & 20.0 & 24.0 & 18.6 & 22.6 \\
\hline Guam & $27.7-29.2$ & $4.3-4.4$ & 21.1 & 24.1 & 20.4 & 23.4 \\
\hline Off Mexico & $22.5-28.0$ & $4.4-4.5$ & 17.6 & 20.9 & 14.9 & 18.0 \\
\hline $\begin{array}{c}\text { Plant Ship } \\
\text { Pacific }\end{array}$ & $27.1-28.5$ & $4.6-4.8$ & 19.4 & 22.8 & 18.1 & 21.7 \\
\hline Ivory Coast & $24.3-28.1$ & 4.5 & 19.2 & 22.1 & 16.8 & 19.7 \\
\hline
\end{tabular}


SUMMARY OF SITE PARAMETERS

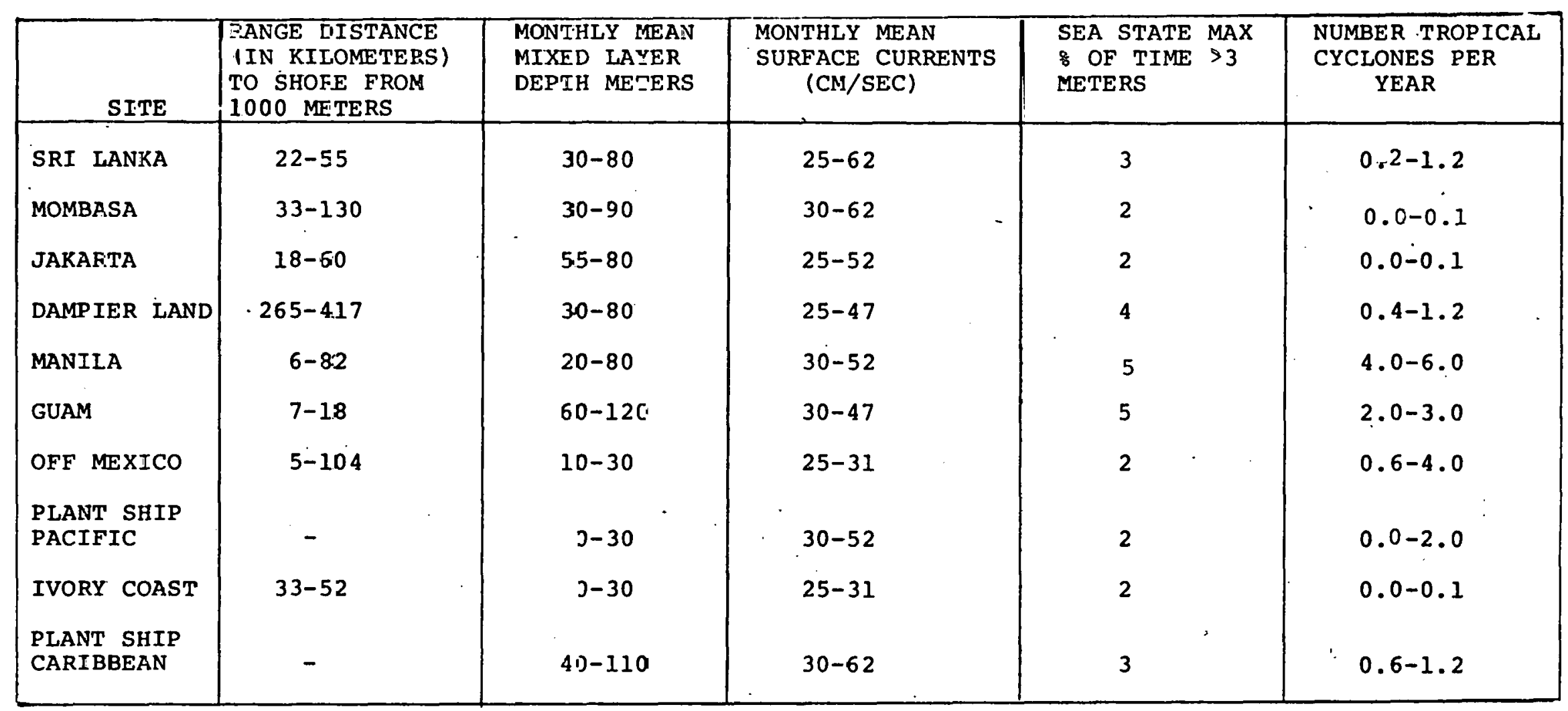


The coastal waters off Mombassa, Kenya were selected for study for their potential for Ocean Thermal Energy Conversion (OTEC) use. The area examined is located in the southwestern equatorial region. The data file was searched between $40^{\circ}-45^{\circ}$ East longitude and between $5^{\circ}$ South latitude and $3^{\circ}$ North latitude. The area south of the equator was. examined separately from the area north of the equator. A usable thermal resource exists for both the northern and southern sections examined.

The Mombassa site compares unfavorably with most of the other 10 sites studied under this contract if $\Delta T$ is used as the only criteria. An annual average $\Delta \mathrm{T}$ of $20^{\circ} \mathrm{C}$ is not reached until a depth of 1100 meters in the northern section, and at 1000 meters in the southern section. The average of the monthly mean $\Delta \mathrm{Ts}$ at 500 meters is $17.0^{\circ} \mathrm{C}$ in the north section and $17.6^{\circ} \mathrm{C}$ in the southern section. The thermal resource is definitely better in the southern position. There is a mixed layer throughout the year that is advantageous for OTEC development. Winds and storms are not a problem for the site. Low sea and swell cunditions are characteristic. Surface current conditions are fairly complicated. The distance from shore to the 1000 meter depth varies, depending on what latitude is chosen for the site. One thousand meter depths are between 30 and 130 kilometers $(\approx 18$ and 70 nautical miles) from land. Off the city of Mombassa itself, the distance is 111 kilometers (60 
nautical miles). Thus, although the thermal resource is. better in the southern section, access to shore is better for the northern section. 


\section{BÄTHYMETRY}

The general location of the prospective site is shown in Figure II-1. Figure II-2 provides the rough bathymetry of the area taken the U.S Naval Oceanographic Office, 1974, [39]. The width of the continental shelf is more uniform in the northern section than the southern. The steepness of the continental slope is also less variable for the area north of the equator. Off most of the coast in consideration the continental shelf is neither extremely wide as in possible OTEC sites in the GuIf of Mexico nor very narrow as the shelf is off puerto Rico for example. The shelf is most narrow for this East African site near the equator. The steepest continental slope for this site is also near the equator. South of $2^{\circ} \mathrm{S}$ latitude the shelf becomes more extensive and the slope less steep. Thus the distance from the port city of Mombasa, Kenya at $04^{\circ} 02^{\prime}$ 's latitude and $39^{\circ}$. $37 ' E$ longitude to the outer limit of the continental shelf is 38 kilometers ( 20.5 nautical miles), and the distance to water 1000 meters deep is approximately 111 kilometers (60 nautical miles).

The extreme distances for each section from shore to depths of interest are shown in Table II-l. 


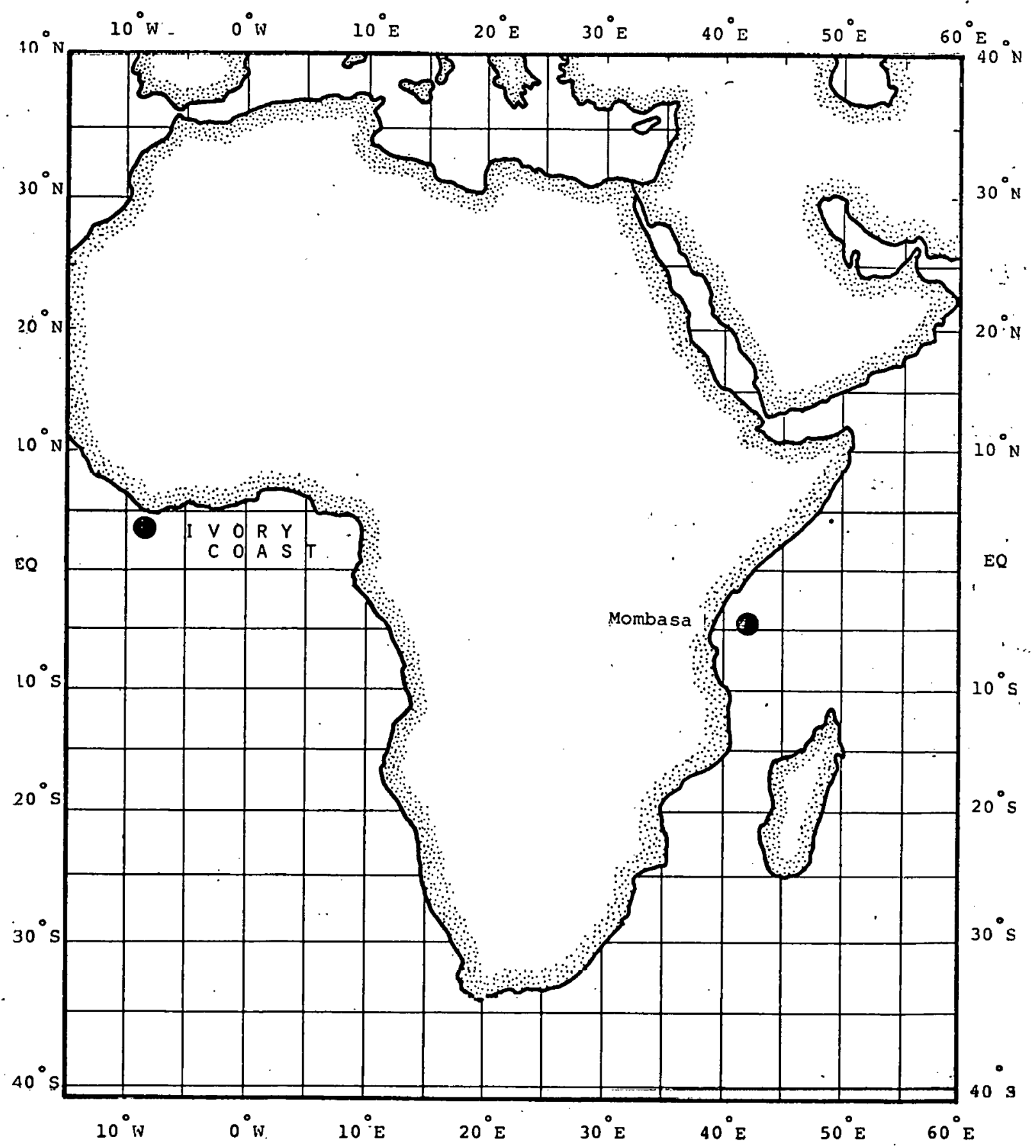

FIGURE II-1: LOCATOR CHART SHOWING THF TOC.ATTON OF THE MOMBASA SITE 


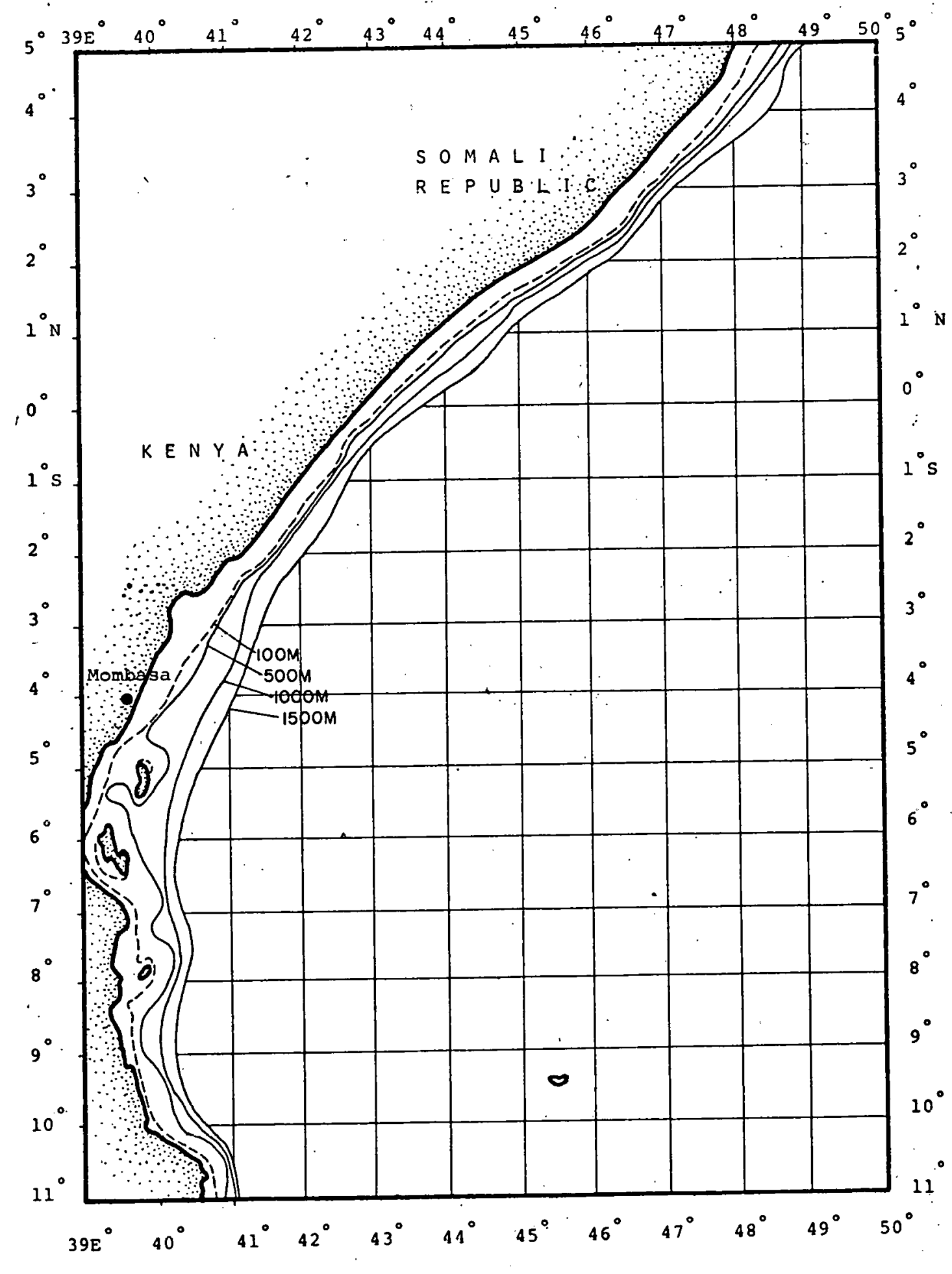

FIGURE II-2: CHART SHOWING ROUGH BATHYMETRY TO 1500 METERS OFF MOMBASA. (From U.S. Naval Oceanographic Office, 1974, [39].) 
TABLE II-I: DISTANCES TO SELECTED DEPTHS OFF MOMBASA .

North Section $0^{\circ}-3^{\circ} \cdot \mathrm{N} / 40-45^{\circ} \mathrm{E}$

\begin{tabular}{|c|c|c|c|}
\hline $\begin{array}{c}\text { DEPTH } \\
\text { (Meters) }\end{array}$ & $\begin{array}{c}\text { CLOSEST DISTANCE } \\
\text { (Kilometers) }\end{array}$ & . & $\begin{array}{c}\text { FARTHEST DISTANCE } \\
(\text { Kilometers })\end{array}$ \\
\hline 100 & 16.7 & & .37 \\
\hline 500 & 25.9 & & 50 \\
\hline 1000 & 33.3 & & 56 \\
\hline 1500 & 52.0 &. & 78 \\
\hline
\end{tabular}

South Section $0^{\circ}-5^{\circ} \mathrm{S} / 40-45^{\circ} \mathrm{E}$

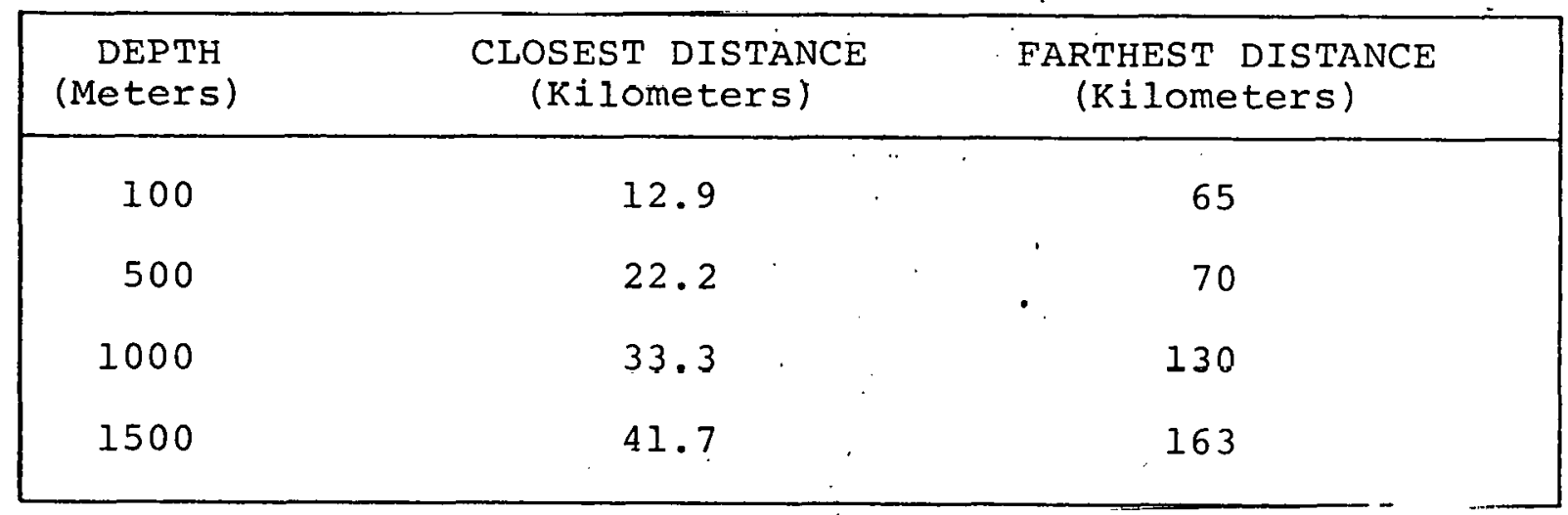

City of Mombasa

\begin{tabular}{|cccc|}
\hline $\begin{array}{c}\text { DEY'H } \\
\text { (Meters) }\end{array}$ & $\begin{array}{c}\text { CLOSEST DISTANCE } \\
\text { (Kilometers) }\end{array}$ & $\begin{array}{c}\text { FARTHEST DISTANCE } \\
\text { (Kilometers) }\end{array}$ \\
\hline 100 & 38.0 & $\ddots$ & - \\
500 & 43.5 & $\ddots$ & - \\
1000 & 111.0 & $\ddots$ & - \\
1500 & 146.0 & & \\
\hline
\end{tabular}


III: THERMAL RESOURCE

Tables III-1 ard III-2 provide the monthly most probable temperature profiles as a function of depth for the northern and southern sections: Tables III-3 and III-4 shows the same data tabulated in terms off $\Delta \mathrm{T}$, the surface temperature minus the temperature at depth differential ( $\left.{ }^{\circ} \mathrm{C}\right)$ by month. There is data coverage for all the standard depths for both the northern and southern sections studied. The main sources for the temperature data were NOAA's National Oceanographic Data Center, and the'U.S. Navy's Fleet Numerical Weather Central, although soundings from other sources were added when available. There is also data for each month of the year, although not at each depth. When the available data was plotted some month-to-month variability was present which was caused by the sparse and non-uniform data sample rather than by real variations in the ocean. These short-period time variations were removed by a filtering process described by wolff, et al (1977)! [44]. This historical oceanographic data shows that the temperature profile off Mombassa is usable for OTEC exploitation. The thermoclinc of this area is shallower and thinner than the thermocline in areas south or east of this site. The temperature differences are less within the thermocline in the western Indian Ocean.

The temperatures at depths for the western Indian Ocean are generally warmer than those in the Atlantic or Pacific. The mean annual temperatures for the southern and northern 
TABLE III-1: MONTHLY MOST PROBABLE TEMPERATURE $\left({ }^{\circ} \mathrm{C}\right)$ PROFILE MOMBASA (NORTH SECTION) $0-3^{\circ} \mathrm{N} / 40-45^{\circ} \mathrm{E}$

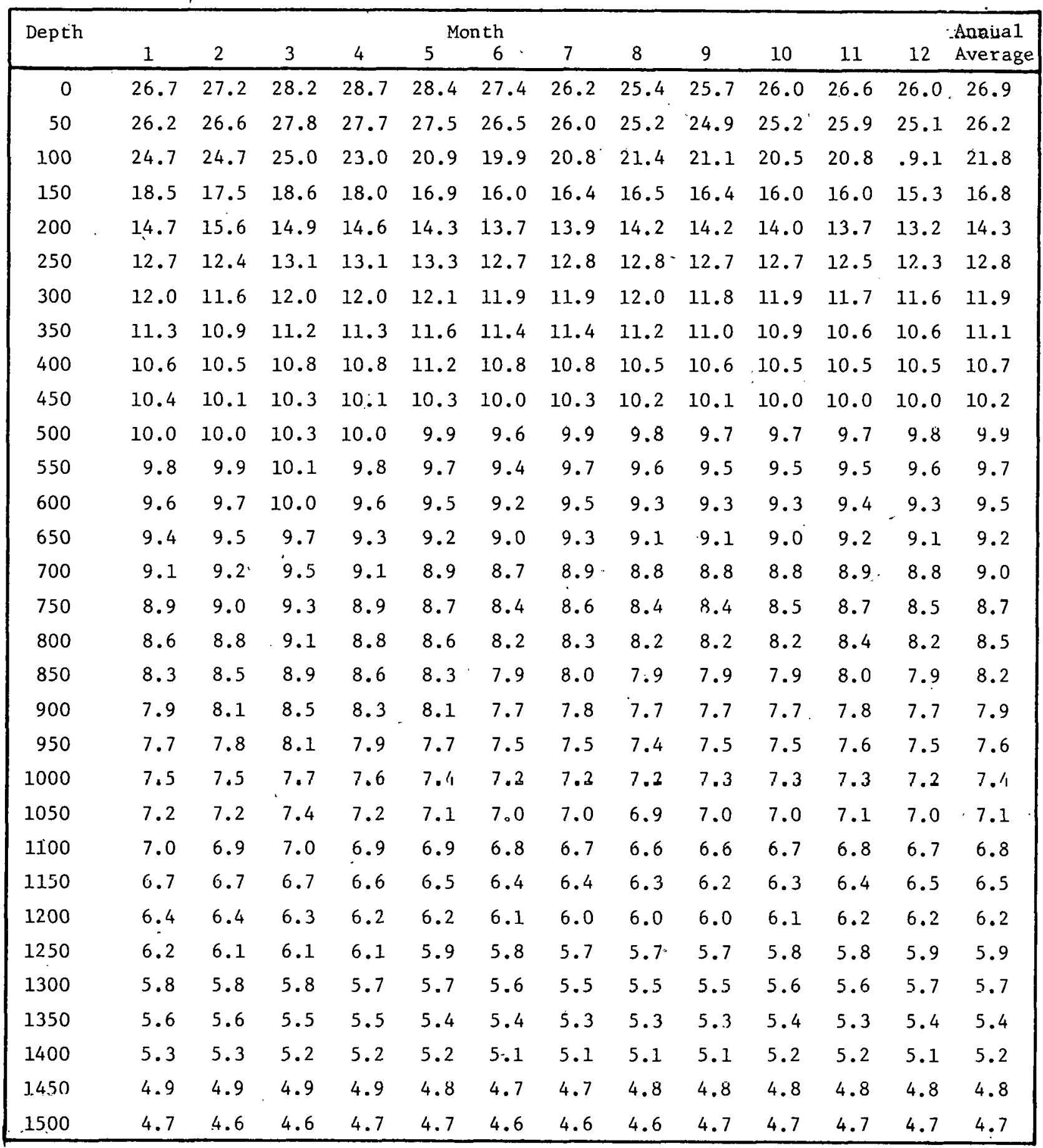


TABI.E III-2: MONTHLY MOST PROBABLE TEMPERATURE $\left({ }^{\circ} \mathrm{C}\right)$ PROFILE MOMBASA (SOUTH SECTION) $0-5^{\circ} \mathrm{S} / 40-45^{\circ} \mathrm{E}$

\begin{tabular}{|c|c|c|c|c|c|c|c|c|c|c|c|c|c|}
\hline Depth & 1 & 2 & 3 & 4 & 5 & $\begin{array}{c}\text { Month } \\
6\end{array}$ & 7 & 8 & 9 & 10 & 11 & 12 & $\begin{array}{c}\text { Annual } \\
\text { Mean }\end{array}$ \\
\hline 0 & 27.0 & 27.3 & 27.7 & 28.4 & 28.0 & 27.2 & 26.2 & 25.5 & 25.6 & 26.0 & 26.8 & 27.1 & 26.9 \\
\hline 50 & 26.2 & 26.3 & 26.6 & 26.9 & 26.9 & 26.5 & 25.8 & 25.1 & 25.0 & 25.2 & 25.7 & 26.0 & 26.0 \\
\hline 100 & 21.7 & 22.6 & 22.8 & 22.0 & $21: 2$ & 20.8 & 21.5 & 21.5 & 22.1 & 22.1 & 21.9 & .21 .5 & 21.8 \\
\hline 150 & 16.9 & 17.7 & 18.1 & 17.0 & 16.2 & 15.8 & 16.3 & 16.5 & 16.6 & 16.6 & 16.4 & 16.3 & 16.7 \\
\hline 200 & 13.7 & 13.9 & 14.2 & 14.0 & 13.6 & 13.4 & 13.7 & 14.2 & 14.2 & 14.2 & 13.9 & 13.8 & 13.9 \\
\hline 250 & 12.3 & 12.3 & 12.6 & 12.4 & $12: 2$ & 12.0 & 12.3 & 12.8 & 12.7 & 12.7 & 12.4 & 12.4 & 12.4 \\
\hline 300 & 11.3 & 11.3 & 11.5 & 11.4 & 11.2 & 11.2 & 11.4 & 11.8 & 11.7 & 11.6 & 11.4 & 11.3 & 11.4 \\
\hline 350 & 10.7 & 10.7 & 10.9 & 10.8 & 10.5 & 10.5 & 10.7 & 10.9 & 10.8 & 10.6 & 10.6 & 10.5 & 10.7 \\
\hline 400 & 10.1 & 10.1 & 10.3 & 10.2 & 10.1 & 10.1 & 10.2 & 10.3 & 10.3 & 9.9 & 9.9 & 9.8 & 10.1 \\
\hline 450 & 9.8 & 9.8 & 9.9 & 9.8 & 9.7 & 9.6 & 9.7 & 9.8 & 9.8 & 9.4 & 9.5 & 9.4 & 9.7 \\
\hline 500 & 9.4 & 9.3 & 9.2 & 9.2 & 9.2 & 9.2 & 9.4 & 9.5 & 9.7 & 9.1 & 9.1 & 8.9 & 9.3 \\
\hline 550 & 9.2 & 9.2 & 9.1 & 9.0 & 8.9 & 8.8 & 9.0 & 9.1 & 9.2 & 8.6 & 8.6 & 8.7 & 9.0 \\
\hline 600 & 8.9 & 9.0 & 8.9 & 8.8 & 8.6 & 8.6 & 8.8 & 8.9 & 8.9 & 8.3 & 8.3 & 8.3 & 8.7 \\
\hline 650 & 8.7 & 8.8 & 8.7 & 8.6 & 8.4 & 8.4 & 8.5 & 8.6 & 8.7 & 8.2 & 8.2 & 8.1 & 8.5 \\
\hline 700 & 8.4 & 8.4 & 8.3 & 8.2 & 8.2 & 8.3 & 8.3 & 8.4 & 8.4 & 8.0 & 7.9 & 8.0 & 8.2 \\
\hline 750 & 8.1 & 8.1 & 8.0 & 7.9 & 7.9 & 8.1 & 8.2 & 8.2 & 8.1 & 7.8 & 7.0 & 8.1 & 8.0 \\
\hline 800 & 7.9 & 7.9 & 7.7 & 7.7 & 7.6 & 7.8 & 7.8 & 7.9 & 7.8 & 7.9 & 7.5 & 7.6 & 7.8 \\
\hline 850 & 7.7 & 7.8 & 7.5 & 7.4 & 7.4 & 7.6 & 7.6 & 7.7 & 7.6 & 7.4 & 7.3 & 7.7 & 7.6 \\
\hline 900 & 7.2 & 7.3 & 7.3 & 7.0 & 6.8 & 7.2 & 7.4 & 7.5 & 7.4 & 7.2 & 7.1 & 7.0 & 7.2 \\
\hline 950 & 7.2 & 7.2 & 6.8 & 6.6 & 6.6 & 7.0 & 7.2 & 7.3 & 7.1 & 6.9 & 6.8 & 7.0 & 7.0 \\
\hline 1000 & 6.9 & 6.9 & 6.5 & 6.4 & 6.3 & 6.7 & 6.9 & 7.0 & 6.9 & 6.7 & 6.6 & 6.8 & 6.7 \\
\hline 1050 & 6.7 & 6.7 & 6.3 & 6.1 & 6.0 & 6.4 & 6.7 & 6.8 & 6.7 & 6.4 & 6.4 & 6.5 & 6.5 \\
\hline 1100 & 6.5 & 6.1 & 6.1 & 5.9 & 5.8 & 6.2 & 6.4 & 6.6 & 6.4 & 6.2 & 6.1 & 6.3 & 6.2 \\
\hline 1150 & 6.3 & 6.3 & 5.9 & 5.6 & 5.5 & 5.9 & 6.2 & 6.4 & 6.1 & 5.9 & 5.8 & 6.0 & 6.0 \\
\hline 1200 & 5.9 & 5.9 & 5.6 & 5.4 & 5.4 & 5.7 & 5.9 & 6.1 & 5.9 & 5.6 & 5.5 & 5.7 & 5.7 \\
\hline 1250 & 5.7 & 5.7 & 5.4 & 5.2 & 5.1 & 5.5 & 5.7 & 5.8 & 5.6 & 5.4 & 5.3 & 5.4 & 5.5 \\
\hline 1300 & 5.4 & 5.4 & 5.2 & 5.0 & 5.0 & 5.2 & 5.4 & 5.5 & 5.3 & 5.1 & 5.1 & 5.2 & 5.2 \\
\hline 1350 & 5.2 & 5.2 & 5.0 & 4.8 & 4.7 & 5.0 & 5.1 & 5.2 & 5.0 & 4.9 & 4.9 & 5.0 & 5.0 \\
\hline 1400 & 4.9 & 4.9 & 4.8 & 4.7 & 4.7 & 4.7 & 4.8 & 4.8 & 4.7 & 4.6 & 4.6 & 4.7 & $4.7^{\prime}$ \\
\hline 1450 & 4.5 & 4.6 & $4.5^{\circ}$ & 4.5 & 4.5 & 4.5 & 4.6 & 4.6 & 4.5 & 4.4 & 4.3 & 4.4 & 4.5 \\
\hline 1500 & 4.4 & 4.3 & 4.2 & 4.2 & 4.2 & 4.3 & 4.3 & 4.2 & 4.2 & 4.1 & 4.2 & 4.3 & 4.2 \\
\hline
\end{tabular}


TABLE III-3: SURFACE TEMPERATURE - TEMPERATURE AT DEPTH DIFFERENTIAL $\left({ }^{\circ} \mathrm{C}\right)$ BY MONTHS MOMBASA (NORTH SECTION) $0-3^{\circ} \mathrm{N} / 40-45^{\circ} \mathrm{E}$

\begin{tabular}{|c|c|c|c|c|c|c|c|c|c|c|c|c|c|}
\hline Depth & 1 & 2 & 3 & 4 & 5 & $\begin{array}{c}\text { Month } \\
6\end{array}$ & 7 & 8 & 9 & 10 & 11 & 12 & $\begin{array}{l}\text { Annual } \\
\text { Mean }\end{array}$ \\
\hline 50 & 0.5 & 0.6 & 0.4 & 1.0 & 0.9 & 0.9 & 0.2 & 0.2 & 0.8 & 0.8 & 0.7 & 0.9 & 0.6 \\
\hline 100 & 2.0 & 2.5 & 3.2 & 5.7 & 7.5 & 7.5 & $5: 4$ & 4.0 & 4.6 & 5.5 & 5.8 & 6.9 & 5.1 \\
\hline 150 & 8.2 & 9.7 & 9.6 & 10.7 & 11.5 & 11.4 & 9.8 & 8.9 & 9.3 & 10.0 & 10.6 & 10.7 & 10.0 \\
\hline 200 & 12.0 & 11.6 & 13.3 & 14.1 & 14.1 & 13.7 & 12.3 & 11.2 & 11.5 & 12.0 & 12.9 & 12.8 & 12.6 \\
\hline 250 & 14.0 & 14.8 & 15.1 & 15.6 & 15.1 & 14.7 & 13.4 & 12,6 & 13.0 & 13.3 & 14.1 & 13.7 & 14.1 \\
\hline 300 & 14.7 & 15.6 & 16.2 & 16.7 & 16.3 & 15.5 & 14.3 & $13: 4$ & 13.9 & 14.1 & 14.9 & 14.4 & 15.0 \\
\hline 350 & 15.4 & 16.3 & 17.0 & 17.4 & 16.8 & 16.0 & 14.8 & 14.2 & 14.7 & 15.1 & 16.0 & 15.4 & 15.8 \\
\hline $400^{\circ}$ & $16: 1$ & 16.7 & 17.4 & 17.9 & 17.2 & 16.6 & 15.4 & 14.9 & 15.1 & 15.5 & 16.1 & 15.5 & 16.2 \\
\hline 450 & 16.3 & 17.1 & 17.9 & 18.6 & 18.1 & 17.4 & 15.9 & 15.2 & 15.6 & 16.0 & 16.6 & 16.0 & 16.7 \\
\hline 500 & 16.7 & 17.2 & 17.9 & 18.7 & 18.5 & $17.8^{\circ}$ & 16.3 & 15.6 & 16.0 & 16.3 & 16.9 & 16.2 & 17.0 \\
\hline 550 & 16.9 & 17.3 & 18.1 & 18.9 & 18.7 & 18.0 & 16.5 & 15.8 & 16.2 & 16.5 & 17.1 & 16.4 & 17.2 \\
\hline 600 & 17.1 & 17.5 & 18.2 & 19.1 & 18.9 & 18.2 & 16.7 & 16.1 & 16.4 & 16.7 & 17.2 & 16.7 & 17.4 \\
\hline 650 & 17.3 & 17.7 & 18.5 & 19.1 & 19.2 & 18.1 & 16.9 & 16.3 & 16.6 & 17.0 & 17.1 & 16.9 & 17.6 \\
\hline 700 & 17.6 & 18.0 & 18.7 & 19.6 & 19.5 & 18.7 & 17.3 & 16.6 & 16.9 & 17.2 & 17.7 & 17.2 & 17.9 \\
\hline 750 & 17.8 & 18.2 & 18.9 & 19.8 & 19.7 & 19.0 & 17.6 & 17.0 & 17.3 & 17.5 & 17.9 & 17.5 & 18.2 \\
\hline 800 & 18.1 & 18.4 & 19.1 & 19.9 & 19.8 & 19.2 & 17.9 & 17.2 & 17.5 & 17.8 & 18.2 & 17.8 & 18.4 \\
\hline 850 & 18.4 & 18.7 & 19.3 & 20.1 & 20.1 & 19.5 & 18.2 & 17.5 & 17.8 & 18.1 & 18.6 & 18.1 & 18.7 \\
\hline 900 & 18.8 & 19.1 & 19.7 & 20.4 & 20.3 & 19.7 & 18.4 & 17.7 & 18.0 & 18.3 & 18.8 & 18.3 & 19.0 \\
\hline 950 & 19.0 & 19.4 & 20.1 & 20.8 & 20.7 & 19.9 & 18.7 & 18.0 & 18.2 & 18.5 & 19.0 & 18.5 & 19.2 \\
\hline 1000 & 19.2 & 19.7 & 20.5 & 21.1 & 21.0 & 20.2 & 19.0 & 18.2 & 18.4 & 18.7 & 19.3 & 18.8 & 19.5 \\
\hline 1050 & 19.5 & 20.0 & 20.8 & 21.5 & 21.3 & 20.4 & 19.2 & 18.5 & 18.7 & 19.0 & 19.5 & 19.0 & 19.8 \\
\hline 1100 & 19.7 & 20.3 & 21.2 & 21.8 & 21.5 & 20.6 & 19.5 & 18.8 & 19.1 & 19.3 & 19.8 & 19.3 & 20.1 \\
\hline 1150 & 20.0 & 20.5 & 21.5 & 22.1 & 21.9 & 21.0 & 19.8 & 19. 1 & 19.5 & 19.7 & 20.2 & 19.5 & 20.4 \\
\hline 1200 & 20.3 & 20.8 & 21.4 & $\angle L .5$ & $\angle L . \angle$ & 21.3 & 211.2 & $1 \dot{y} .4$ & 19.7 & 19.9 & 20.4 & 19.8 & 20.7 \\
\hline 1250 & 20.5 & $21 . .1$ & 22.1 & 22.6 & 22.5 & 21.6 & 20.5 & 19.7 & 20.0 & 20.2 & 20.8 & 20.1 & 21.0 \\
\hline 1300 & 20.9 & 21.4 & 22.4 & 23.0 & 22.7 & 21.8 & 20.7 & 19.9 & 20.2 & 20.4 & 21.0 & 20.3 & 21.2 \\
\hline 1350 & 21.1 & 21.6 & 22.7 & 23.2 & 23.0 & 22.0 & 20.9 & 20.1 & 20.4 & 20.6 & 21.3 & 20.6 & 21.5 \\
\hline 1400 & 21.4 & 21.9 & 23.0 & 23.5 & 23.2 & 22.3 & 22.1 & 20.3 & 20.6 & 20.8 & 21.4 & 20.9 & 21.8 \\
\hline .1450 & 21.8 & 22.3 & 23.3 & 23.8 & 23.6 & 22.7 & 21.5 & 20.6 & 20.9 & 21.2 & 21.8 & 21.2 & 22.0 \\
\hline 1500 & 22.0 & 22.6 & 23.6 & 24.0 & 23.7 & 22.8 & 21.6 & 20.8 & 21.0 & 21.3 & 21.9 & 21.3 & $\overline{2} \dot{2} . \overline{2}$ \\
\hline
\end{tabular}


TABLE III-4: SURFACE TEMPERATURE - TEMPERATURE AT DEPTH DIFFERENTIAL $\left({ }^{\circ} \mathrm{C}\right.$ ) BY MONTHS MOMBASA (SOUTH SECTION) $0-5^{\circ} \mathrm{S} / 40-45^{\circ} \mathrm{E}$

\begin{tabular}{|c|c|c|c|c|c|c|c|c|c|c|c|c|c|}
\hline \multirow[t]{2}{*}{ Depth } & \multicolumn{11}{|c|}{ Month } & \multicolumn{2}{|r|}{ Annual } \\
\hline & 1 & 2 & 3 & 4 & 5 & 6 & 7 & 8 & 9 & 10 & 11 & 12 & Mean \\
\hline 50 & 0.8 & 1.0 & 1.1 & 1.5 & 1.1 & 0.7 & 0.4 & 0.4 & 0.6 & 0.8 & 1.1 & 1.1 & 0.9 \\
\hline 100 & 5.3 & 4.7 & 4.9 & 6.4 & 6.8 & 6.4 & 4.7 & 4.0 & 3.5 & 3.9 & 4.9 & 5.6 & 5.1 \\
\hline 150 & 10.1 & 9.6 & 9.6 & 11.4 & 11.8 & 11.4 & 9.9 & 9.0 & 9.0 & 9.4 & 10.4 & 10.8 & 10.2 \\
\hline 200 & 13.3 & 13.4 & 13.5 & 14.4 & 14.4 & 13.8 & 12.5 & 11.3 & 11.4 & 11.8 & 12.9 & 13.3 & 11.9 \\
\hline 250 & 14.7 & 15.0 & 15.1 & 16.0 & 15.8 & 15.2 & 13.9 & 12.7 & 12.9 & 13.3 & 14.4 & 14.7 & 14.5 \\
\hline 300 & 15.7 & 16.0 & 16.2 & 17.0 & 16.8 & 16.0 & 14.8 & 13.7 & 13.9 & 14.4 & 15.4 & 15.8 & 15.5 \\
\hline 350 & 16.3 & 16.6 & 16.8 & 17.6 & 17.5 & 16.7 & 15.5 & 14.6 & $14.8^{\circ}$ & .15 .4 & 16.2 & 16.6 & 16.2 \\
\hline 400 & 16.9 & 17.2 & 17.4 & 18.2 & 17.9 & 17.1 & 16.0 & 15.2 & 15.3 & 16.1 & 16.9 & 17.3 & 16.8 \\
\hline 450 & 17.2 & 17.5 & 17.8 & 18.6 & 18.3 & 27.6 & 16.5 & .75 .7 & 1.5 .8 & 16.6 & 17.3 & 17.7 & 17.2 \\
\hline 500 & 17.6 & 18.0 & 18.5 & 19.2 & 18.8 & 18.0 & 16.8 & i6. 0 & 15.9 & 16.9 & 17.7 & 18.2 & 17.6 \\
\hline 550 & 17.8 & 18.1 & 18.6 & 19.4 & 19.1 & 18.4 & 17.2 & 16.4 & 16.4 & 17.4 & 18.2 & 18.4 & 18.0 \\
\hline 600 & 18.1 & 18.3 & 18.8 & 19.6 & 19.4 & 18.6 & 17.4 & 16.6 & 16.7 & 17.7 & 18.5 & 18.8 & 18.2 \\
\hline 650 & 18.3 & 18.5 & 19.0 & 19.8 & 19.6 & 18.8 & 17.7 & 16.9 & 16.9 & 17.8 & 18.6 & 19.0 & 18.4 \\
\hline 700 & 18.6 & 18.9 & 19.4 & 20.2 & 19.8 & 18.9 & 17.9 & 17.1 & 17.2 & 18.0 & 18.9 & 19.1 & 18.7 \\
\hline 750 & 18.9 & 19.2 & 19.7 & 20.5 & 20.1 & 19.1 & 18.0 & 17.3 & 17.5 & 18.2 & 19.1 & 19.2 & 18.9 \\
\hline 800 & 19.1 & 19.4 & 20.0 & 20.7 & 20.4 & 19.4 & 18.4 & 17.6 & 17.8 & 18.1 & 19.3 & 19.5 & 19.1 \\
\hline 850 & 19.3 & 19.5 & 20.2 & 21.0 & 20.6 & 19.6 & 18.6 & 17.8 & 18.0 & $18.6^{\circ}$ & 19.5 & 19.6 & 19.4 \\
\hline 900 & 19.8 & 20.0 & 20.4 & 21.4 & 21.2 & 20.0 & 18.8 & 18.0 & 18.2 & 18.8 & 19.7 & 20.1 & 19.7 \\
\hline 950 & 19.8 & 20.1 & 20.9 & 21.8 & 21.4 & 20.2 & 19.0 & 18.2 & 18.5 & 19.1 & 20.0 & 20.1 & 19.9 \\
\hline 1000 & 20.1 & 20.4 & 21.2 & 22.0 & 21.7 & 20.5 & 19.3 & 18.5 & 18.7 & 19.3 & 20.2 & 20.3 & 20.2 \\
\hline 1050 & 20.3 & 20.6 & 21.4 & 22.3 & 22.0 & 20.8 & 19.5 & 18.7 & 18.9 & 19.6 & 20.4 & 20.6 & 20.4 \\
\hline 1100 & 20.5 & 21.2 & 21.6 & 22.5 & 22.2 & 21.0 & 19.8 & 18.9 & 19.2 & 19.8 & 20.7 & 20.8 & 20.7 \\
\hline 1150 & 20.7 & 21.0 & 21.8 & 22.8 & 22.5 & 21.3 & 20.0 & 19.1 & 19.5 & 20.1 & 21.0 & 21.1 & 20.9 \\
\hline 1200 & 21.1 & 21.4 & 22.1 & 23.0 & $22: 6$ & $21 . .5$ & 20.3 & 19.4 & 19.7 & 20.4 & 21.3 & 21.4 & 21.2 \\
\hline 1250 & 21.3 & 21.6 & 22.3 & 23.2 & .22 .9 & 21.7 & 20.5 & 19.7 & 20.0 & 20.6 & 21.5 & 21.7 & 21.4 \\
\hline 1300 & 21.6 & 21.9 & 22.5 & 23.4 & 23.0 & 22.0 & 20.8 & 20.0 & 20.3 & 20.9 & 21.7 & 21.9 & 21.7 \\
\hline 1350 & 21.8 & 22.1 & 22.7 & 23.6 & 23.3 & 22.2 & 21.1 & 20.3 & 20.6 & 21.1 & $21 . \dot{y}$ & 22.1 & 21.9 \\
\hline 1400 & 22.1 & 22.4 & 22.9 & 23.7 & 23.3 & 22.5 & 21.4 & 20.7 & 20.9 & 21.4 & 22.2 & 22.4 & 22.2 \\
\hline 1450 & 22.5 & 22.7 & 23.2 & 23.9 & 23.5 & 22.7 & 21.6 & 20.9 & 21.1 & 21.6 & 22.5 & 22.7 & 22.4 \\
\hline 1500 & 22.6 & 23.0 & 23.5 & 24.2 & 23.8 & 22.9 & 21.9 & 21.3 & 21.4 & 21.9 & 22.6 & 22.8 & 22.7 \\
\hline
\end{tabular}


sections of the Mombassa region at 1000 meters are $6.7{ }^{\circ} \mathrm{C}$ and $7.4^{\circ} \mathrm{C}$, respectively. For representative sites in the Pacific. and Atlantic such as Manila and the Ivory Coast, the mean annual temperature at 1000 meters is $4.5^{\circ} \mathrm{C}$. The monthly mean surface temperatures, however, are not any warmer than sites in other oceans, for either Mombassa section, ranging between $25.1^{\circ} \mathrm{C}$ and $29.1^{\circ} \mathrm{C}$. Annual average $\Delta \mathrm{T}$ s are therefore somewhat less than most of the other sites being examined, under this contract. This is particularly true of the northern site where the annual mean $\Delta \mathrm{T}$ at 1000 meters is $19.5^{\circ} \mathrm{C}$. At 1000 meters, the annual mean $\Delta \mathrm{T}$ for the southern site is $20.2^{\circ} \mathrm{C}$. At 500 meters, the annual average of monthly mean $\Delta \mathrm{Ts}$ is $17.0^{\circ} \mathrm{C}$ for the northern site and $17.6^{\circ} \mathrm{C}$ for the southern section with a monthly mean temperature as low as $15.6^{\circ} \mathrm{C}$ during the coldest month.

Figure III-1 shows monthly $\Delta \mathrm{T}$ contours plotted versus depth for the northern section." Figure III-2 provides a plot of monthly $\Delta \mathrm{T}$ coutours for the southern section. 


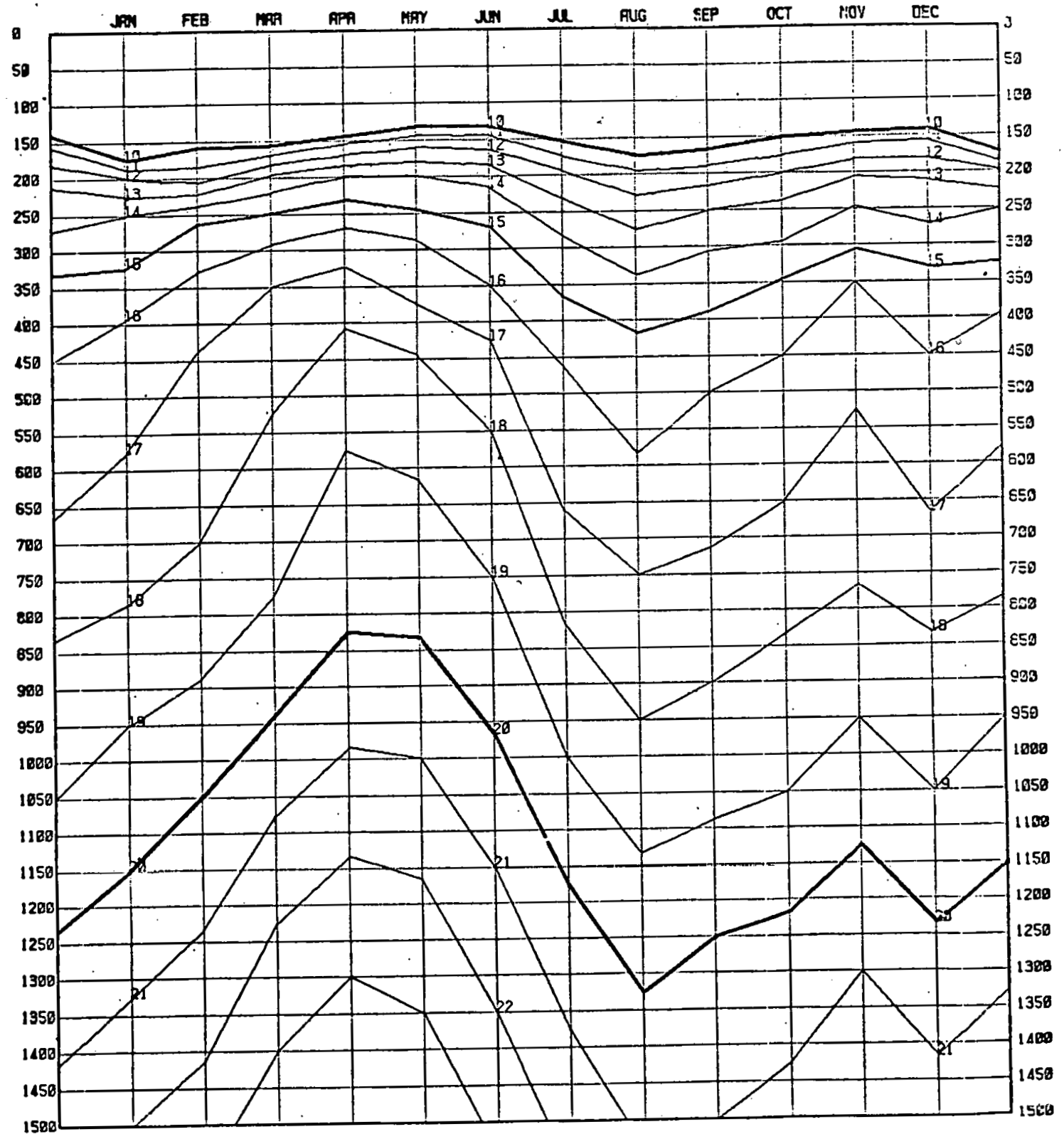

FIGURE III-1: CHART SHOWING MONTHLY $\triangle T$ CONTOURS $\left(C^{\circ}\right)$ AT A DEPTH RESOLUTION OF 50 METERS FOR MOMBASA (NORTH SECTION) $0-3 \mathrm{~N} / 40-45 \mathrm{E}$ 


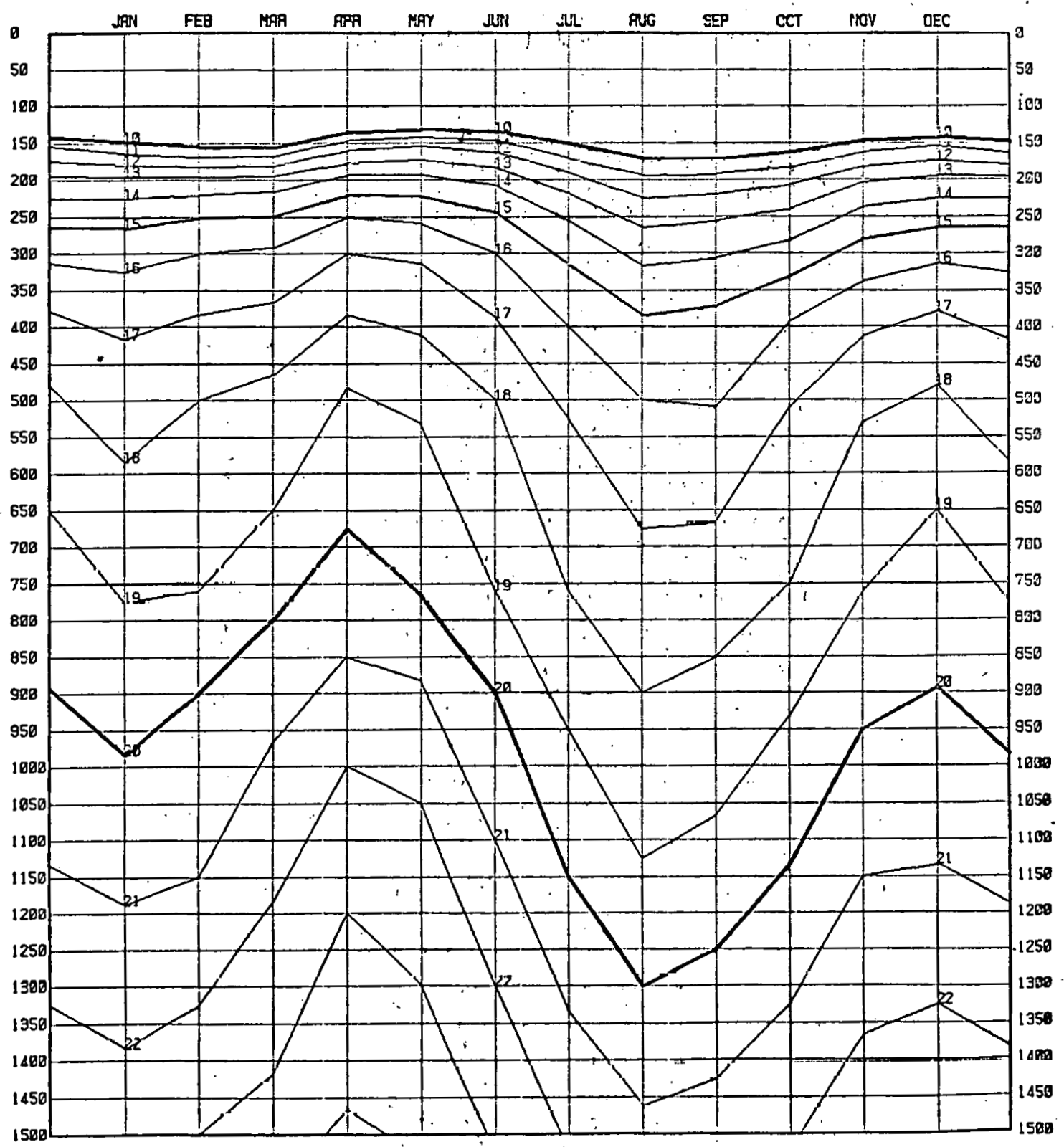

FTGTRE III=2: CHART SHOWING MONTIILY $\triangle T$ CONTOURS $\left({ }^{\circ} \mathrm{C}\right)$ AT A DEPTH RESOLUTION OF 50 METERS FOP. MOMBISA (EOUTII GECTION) $0-55 / 40-45^{\circ} \mathrm{E}$ 
IV. MIXED LAYER DEPTH

For OTEC purposes, the upper mixed layer is defined as the depth at which the temperature is first $1^{\circ} \mathrm{C}$ colder than the sea surface temperature. There is an upper mixed layer depth (MLD) throughout the year both north and south of the equator. The mixed layer depth has a mean of about 50 meters. The values of the MLD range between 30 meters and 90 meters. There is some variation between the northern section and the southern section. Representative values for the mixed layer depth determined from Colburn's The Thermal Structure of the Indian Ocean (1974), [ 6 6], and our own temperature data file are shown in Table IV-l.

TABLE IV-1: TYPICAL MIXED LAYER DEPTHS (METERS) OFF MOMBASA.

\begin{tabular}{|c|c|c|c|c|c|c|}
\hline & JAN-FEB & MAR-APR & MAY-JUN & JUL-AUG & SEP-OCT & NOV-DEC \\
\hline $\begin{array}{l}\text { Southern } \\
\text { Séction }\end{array}$ & 40 & 40 & 70 & 90 & 70 & 55 \\
\hline $\begin{array}{l}\text { Northern } \\
\text { Section }\end{array}$ & 80 & 50 & 30 & 70 & 70 & 35 \\
\hline
\end{tabular}

A mixed layer depth is helpful for ideal OTEC operating conditions. The MLD off Kenya is deep enough to assure an intake of uniformly warm water. The northern section's MLD during the May-June and November-December periods is not as deep as would be ideal. This MLD is not so deep that recirculation of the mixed exhaust should be a major problem. 
V. WINDS AND STORMS

The northeast monsoons govern the region beginning in November. The transition to the southwest monsoon begins during April and continues in May. Relatively weak winds of 15 knots or less are characteristic of the region. Southeast of Mombasa, there is an offshore wind maxima with occasional observations up to 10.3 meters/second. Still winds over 10.3 meters/second occur infrequently. Typical winds for the region are:

JAN-FEB MAR-APR MAY-JUN JUL-AUG SEP-OCT NOV-DEC

Resultant wind. direction in degrees from $\mathrm{N}$ $60 \downarrow 110 \uparrow 190 \uparrow \quad 170 \uparrow 170 \uparrow 120 \uparrow$

Resultant wind speed in meters per second

wind steadiness in percent

5.7

2.6

5.7

5.7

6.7

3.1

The source of this information is the Meteorological Atlas of the International Indian Ocean Expedition, (1972), [26].

Table V-I, taken from the U.S. Naval Weather Service

(1974), [35] shows basic climatic information for the vicinity of Mombasa, Kenya.

Tables V-2, through V-5 with data from the U.S. Weather Bureau (1938), [31] provides information on the winds for each of the ton sitcs cxamined under this contract. 
Figure V-1 adapted from H.L. Crutcher and R.G. Quayle (1974), [ 7] shows the preferred annual storm tracks for '. this region. Figure V-2 from the same source provides the average number of tropical cyclones per $5^{\circ}$ square per year. As these figures show tropical storms and hurricanes are not a problem for this site, less than 0.1 storms per year affect either of the Mombasa sites. 
TABLE V-1: CLIMATIC SUMMARY FOR MOMBASA, KENYA (Elevation 54.6 meters) Latitude $04^{\circ} 02^{\prime} \mathrm{s}$, Longitude $39^{\circ} 37^{\prime} \mathrm{E}$. From U.S. Naval Weather Service (1974) [35].

\begin{tabular}{|c|c|c|c|c|c|c|c|c|c|c|c|c|}
\hline \multirow[b]{2}{*}{ PARAMETER } & \multicolumn{12}{|c|}{ MONTH } \\
\hline & JAN & FEB & MAR & APR & MAY & JUN & JUL & AUG & SEP & OCT & NOV & $\mathrm{DEC}$ \\
\hline ABSOLUTE MAX TEMP $\left({ }^{\circ} \mathrm{C}\right)$ & 35 & 36 & 35 & 36 & 33 & 32 & 33 & 31 & 32 & 33 & 34 & 36 \\
\hline AVERAGE MAX TEMP $\left({ }^{\circ} \mathrm{C}\right)$ & 33 & 33 & 34 & 32 & 30 & 29 & 29 & 29 & 30 & 31 & 32 & 33 \\
\hline MEAN TEMP $\left({ }^{\circ} \mathrm{C}\right)$ & 28 & 28 & 29 & 28 & 27 & 26 & 25 & 25 & 26 & 26 & 28 & 28 \\
\hline AVERAGE MIN TEMP $\left({ }^{\circ} \mathrm{C}\right)$ & 24 & 24 & 24 & 24 & 23 & 22 & 21 & 22 & 22 & 23 & 24 & 24 \\
\hline ABSOLUTE MIN TEMP $\left({ }^{\circ} \mathrm{C}\right)$ & 21 & 21 & 22 & 21 & 19 & 16 & 18 & 17 & 18 & 18 & 20 & 21 \\
\hline AVERAGE RAINFALL (MM) & 28 & 15 & 69 & 191 & 318 & $11-9$ & 94 & 61 & 66 & 84 & 99 & 64 \\
\hline MEAN NO. DAYS RAIN & 4 & 2 & 6 & 17 & 19 & 14 & 16 & 15 & 12 & 12 & 11 & 9 \\
\hline MEAN NO.DAYS THUNDERSTORM & $<1$ & 1 & 4 & 4 & $<1$ & 0 & 0 & 0 & 0 & $<1$ & 2 & 3 \\
\hline MEAN NO. DAYS FOG & 0 & 0 & $<0$ & 1 & 1 & 0 & 0 & 0 & $<1$ & 0 & 0 & 0 \\
\hline MEAN RELATIVE HUMIDITY $(\%)$ & 69 & 70 & 71 & 69 & 67 & 61 & 64 & 62 & 64 & 64 & 68 & 69 \\
\hline PREVAILING WIND SPEED & $\mathrm{NE}$ & $\mathrm{E}$ & SE & $S$ & $S$ & $S$ & $S$ & $S$ & $\mathrm{SE}$ & $\mathrm{SE}$ & $\mathrm{SE}$ & NE \\
\hline MEAN WIND SPEED (M/S) & 3.6 & 3.6 & 3.6 & 3.6 & 4.1 & 4.6 & 4.1 & 4.1 & 4.1 & 4.1 & 3.1 & 3.1 \\
\hline
\end{tabular}


TABLE V-2

RESULT'ANT WINDS

Average Wind Velocity in Meters per Sécond

\begin{tabular}{|c|c|c|c|c|}
\hline Location & Dec-Jan-Feb & Mar-Apr-May & Jun-Jul-Aug & Sep-Oct-Nov \\
\hline Ivory Coast & $3.1-4.1$ & $3.1-4.1$ & $4.1-5.1$ & $4.1-5.1$ \\
\hline Mombasa & $5.1-6.2$ & $4.1-5.1$ & $6.2-7.2$ & $4.1-5.1$ \\
\hline Sri Lanka & $\begin{array}{l}4.1-5.1 W \\
5.1-6.2 \mathrm{E}\end{array}$ & $4.1-5.1$ & $\begin{array}{l}6.2-7.2 \mathrm{~W} \\
7.2-8.2 \mathrm{E}\end{array}$ & $5.1-6.2$ \\
\hline Jakarta & 4.1 & $3.1-4.1$ & $5.1-6.2$ & $5.1-6.2$ \\
\hline Dampier Land & $4.1-5.1$ & $4.1-6.2$ & $5.1-6.2$ & $4.1-5.1$ \\
\hline Ph111pp1nes & $5.1-6.2$ & $3.1-4.1$ & 4.1 & $6.2-7.2$ \\
\hline Guant & $5.1-6.2$ & $4.1-5.1$ & $4.1-5.1$ & $4.1-5.1$ \\
\hline Off Mexico & $3.1-4.1$ & $2.1-4.1$ & $2.1-4.1$ & $4.1-5.1$ \\
\hline $\begin{array}{l}\text { Plant Ship } \\
\text { Pacific }\end{array}$ & $3.1-4.1$ & $3.1-4.1$ & $4.1-5.1$ & $4.1-6.2$ \\
\hline $\begin{array}{l}\text { Plant Ship } \\
\text { Caribbean }\end{array}$ & $6.2-7.2$ & $5.1-6.2$ & $6.2-8.2$ & $4.1-5.1$ \\
\hline
\end{tabular}


TABLE V-3

FREQUENCY AND PERCENTAGE OF MODERATE GALES AND STRONGER WINDS,

BEAUFORT FORCE 7 AND HIGHER

( $\geq 14.4$ meters per second)

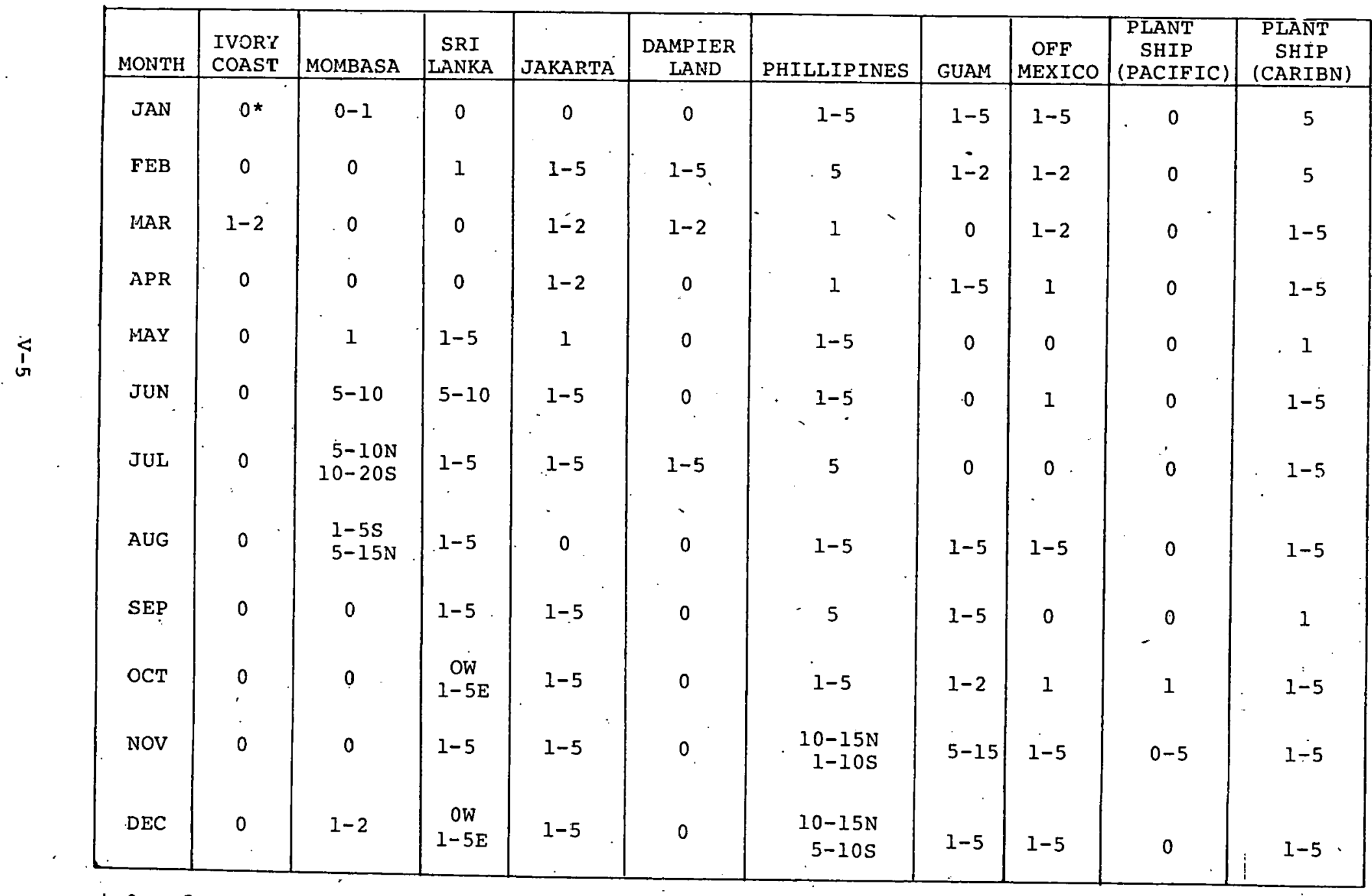

* $0=$ few or none. 
TABLE V-4

PERCENTAGE OF WINDS WITH BEAUFORT FORCE 8 AND HIGHER ( $\geq 17.5$ meters per second)

\begin{tabular}{|c|c|c|c|c|}
\hline LOCATION & Dec-Jan-Feb & Mar-Apr-May & Jun-Jul-Aug & Sep-Oct-Nov \\
\hline Ivory Coast & 0 & 1 & 0 & 0 \\
\hline Mombasa & $\begin{array}{ll}N & 1 \\
S & 0\end{array}$ & 0 & 0 & 0 \\
\hline Sri Lanka & 0 & 0 & 1 , & 0 \\
\hline Jakarta & 1 & 1 & 0 & 1 \\
\hline Dampier Iand & 1 & 1 & 0 & 0 \\
\hline Philippines & 5 & 0 & 1 & $1-5$ \\
\hline Guari & 0 & 1 & 1 & $1-3$ \\
\hline Off Mexico & $i$ & 1 & 0 & 1 \\
\hline $\begin{array}{l}\text { Plant Ship } \\
\text { Pacific }\end{array}$ & 0 & 1 & 0 & 0 \\
\hline $\begin{array}{l}\text { Plant Ship } \\
\text { Caribbean }\end{array}$ & 1 & 0 & 1 & 0 \\
\hline
\end{tabular}


TABLE $V-5$

PREDOMINANT SURFACE WIND DIRECTION

\begin{tabular}{|c|c|c|c|c|c|c|c|c|c|c|}
\hline MONTH & MOMBASA & $\begin{array}{c}\text { SRI } \\
\text { LANKA }\end{array}$ & $\underset{\substack{\text { DAMPIER } \\
\text { LAND }}}{ }$ & JAKARTA & MANILA & GuAM & $\underset{\text { MEXICO }}{\text { OFF }}$ & $\begin{array}{l}\text { PLANT } \\
\text { SHIP } \\
\text { (PACIFIC) }\end{array}$ & $\begin{array}{l}\text { PLANT } \\
\text { SHIP } \\
\text { (CARIBN) }\end{array}$ & $\begin{array}{l}\text { IVORY } \\
\text { COAST }\end{array}$ \\
\hline JAN & $\downarrow$ & $\swarrow$ & $\nearrow$ & $\searrow$ & $\downarrow$ & $\swarrow$ & $\searrow$ & $\swarrow$ & $\swarrow$ & $\uparrow \nearrow$ \\
\hline FEB & $\downarrow$ & $\swarrow$ & $\rightarrow$ & $\rightarrow$ & $\measuredangle$ & $\swarrow$ & $\downarrow$ & $\swarrow$ & $\swarrow$ & $\uparrow$ \\
\hline MAR & $<$ & $\downarrow \circ \zeta$ & $\longrightarrow$ & $\rightarrow$ & $\downarrow$ & $\not$ & $\nu$ & $\swarrow$ & $\leftarrow$ & $\uparrow$ \\
\hline APR & $0^{\circ} \frac{\swarrow}{K}$ & 吗 & $\pi$ & $\kappa$ & $\downarrow$ & $\leftarrow$ & $\nu$ & $\measuredangle$ & $\longleftarrow$ & $\uparrow$ \\
\hline MAY & $\uparrow \uparrow$ & $\nearrow$ & $\pi$ & $k$ & 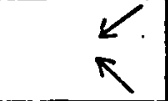 & $\swarrow$ & $\downarrow$ & 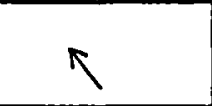 & $\leftarrow$ & $\uparrow$ \\
\hline JUN & $\uparrow$ & $\lambda$ & $\pi$ & $\pi$ & $\nearrow$ & $\leftarrow$ & $\downarrow$ & $y_{i}<$ & $\leftarrow$ & $\uparrow$ \\
\hline JUI & $\uparrow$ & 7 & $\pi$ & $\pi$ & $\pi$ & $\uparrow$ & 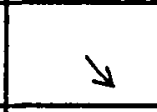 & $\uparrow$ & $\leftarrow$ & $\uparrow$ \\
\hline AUG & $\uparrow$ & $\lambda$ & $\nwarrow$ & $\nwarrow$ & $\nearrow$ & $\leftarrow$ & $\downarrow$ & $\vec{\uparrow} \rightarrow$ & $\leftarrow$ & $\nearrow$ \\
\hline SEP & $\uparrow$ & $\rightarrow 0^{\lambda}$ & $\pi$ & $\pi$ & $\sigma_{7}$ & $\leftarrow$ & $\searrow$ & $\pi$ & $\leftarrow$ & $\uparrow$ \\
\hline ост & $\uparrow$ & $\rightarrow$ & $\pi$ & $\pi$ & $\swarrow$ & $\swarrow$ & $\downarrow$ & $\uparrow$ & $\leftarrow$ & $\uparrow$ \\
\hline Nov & $\frac{\alpha}{K}$ & $\rightarrow \infty$ & $\sqrt{P}$ & $\bar{s}$ & $\swarrow$ & $\downarrow$ & $\downarrow$ & 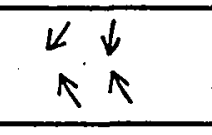 & $\leftarrow$ & $\uparrow$ \\
\hline $\mathrm{DEC}$ & 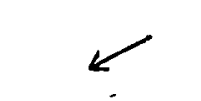 & 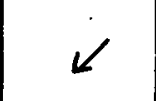 & $\nearrow$ & $r$ & $\downarrow$ & $\swarrow$ & $\downarrow$ & $\leftarrow$ & $<$ & $\nearrow$ \\
\hline
\end{tabular}




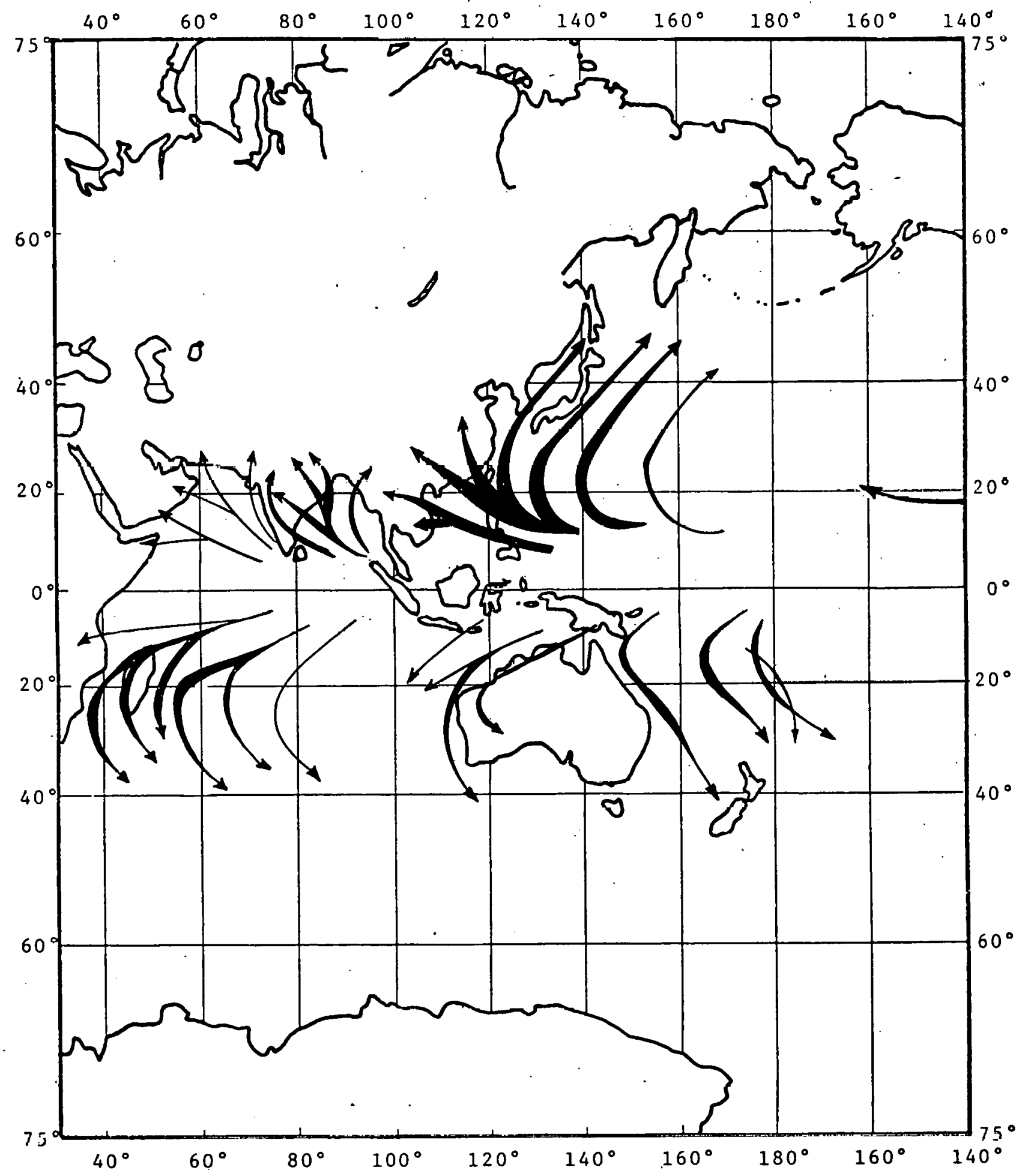

ANNUAL PERFERRED STORM TRACKS FOR TROPICAL STORMS

FIGURE $V-I$

$$
\mathrm{V}-8
$$




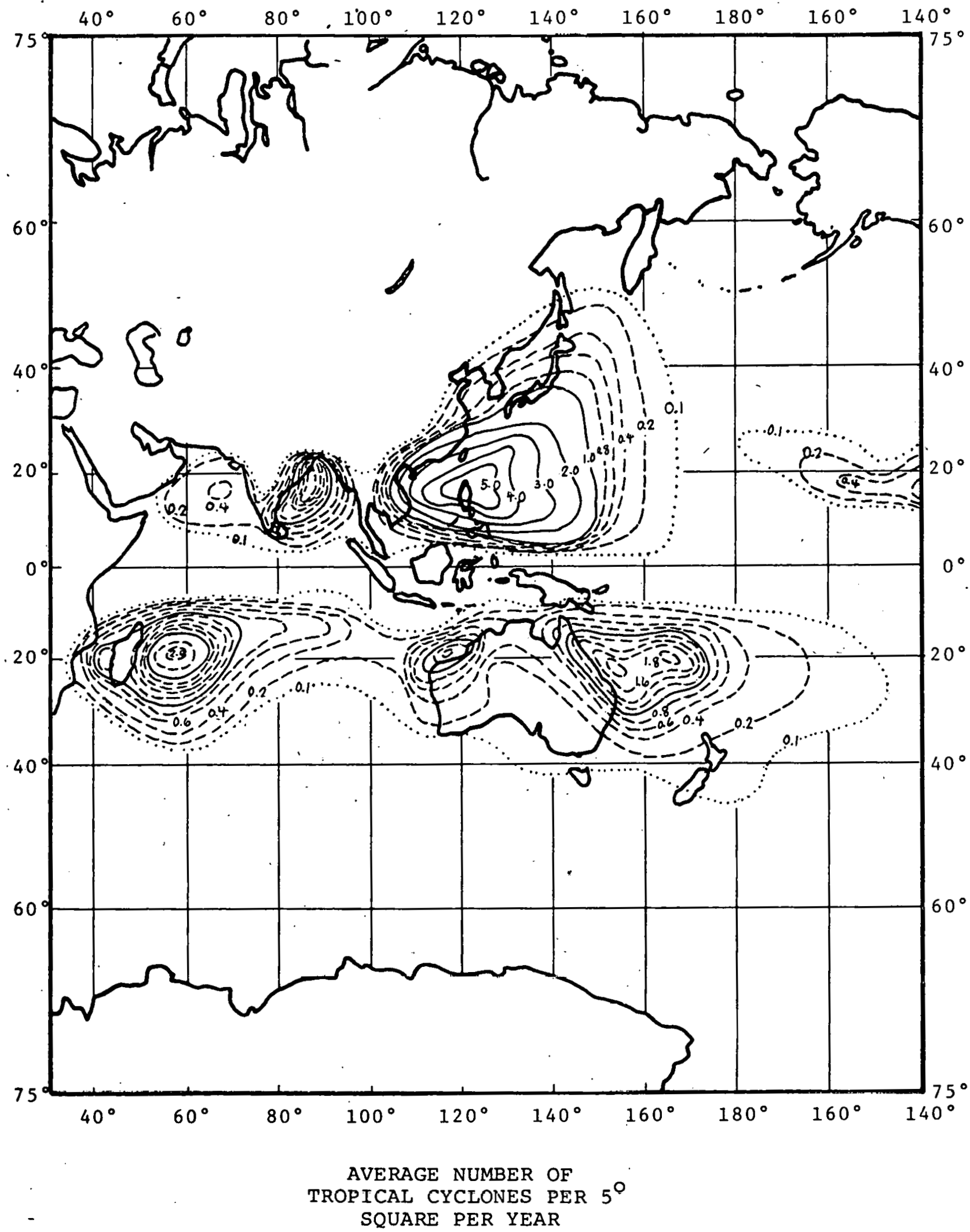

FIGURE $V-2$

$\mathrm{V}-9$ 
VI. SEA AND SWELL CHARACTERISTICS

Sea and swell conditions are generally a response to wind conditions. Since winds off Mombassa are consistently light, it would be expected that high sea and swell conditions are rare. This is indeed the case for the waters off Mombassa. Tables VI-I and Table VI-2 present sea and swell data taken from U.S. Hydrographic office Wind Waves at Sea $(1947,[\underline{5}])$.

TABLE VI-1: PERCENTAGE OF VARIOUS SEA CONDITIONS.

\begin{tabular}{|l|c|c|}
\hline & $\begin{array}{c}\text { Low Seas } \\
(0.3-0.9 \text { meters })\end{array}$ & $\begin{array}{c}\text { High seas } \\
\text { meters or higher })\end{array}$ \\
\hline $\begin{array}{l}\text { JAN-FEB } \\
\text { Northern } \\
\text { Section }\end{array}$ & $20-40 \%$ & $0-10 \%$ \\
\hline $\begin{array}{l}\text { JAN-FEB } \\
\text { Southern } \\
\text { Section }\end{array}$ & $40-80 \%$ & $0-10 \%$ \\
\hline $\begin{array}{l}\text { JuL-AUG } \\
\text { Northern } \\
\text { Section }\end{array}$ & $40-60 \%$ & $0-10 \%$ \\
\hline $\begin{array}{l}\text { JuL-Aug } \\
\text { Solthern } \\
\text { Section }\end{array}$ & $40-60 \%$ & $0-10 \%$ \\
\hline
\end{tabular}


TABLE VI-2: PERCENTAGE OF VARIOUS SWELL CONDITIONS.

\begin{tabular}{|l|c|c|}
\hline & $\begin{array}{c}\text { Low Swell } \\
(0.3-1.8 \text { meters })\end{array}$ & $\begin{array}{c}\text { High Swell } \\
\text { meters or higher })\end{array}$ \\
\hline $\begin{array}{l}\text { JAN-FEB } \\
\text { Northern } \\
\text { Section }\end{array}$ & $40-60 \%$ & $0-10 \%$ \\
\hline $\begin{array}{l}\text { JAN-FEB } \\
\text { Southern } \\
\text { Section }\end{array}$ & $60-80 \%$ & $0-10 \%$ \\
\hline $\begin{array}{l}\text { JuL-AuG } \\
\text { Northern } \\
\text { Section }\end{array}$ & $-40-60 \%$ & $10-20 \%$ \\
\hline $\begin{array}{l}\text { JuL-Aug } \\
\text { Southern } \\
\text { Section }\end{array}$ & $40-60 \%$ & $0-10 \%$ \\
\hline
\end{tabular}

Table VI-3, taken from Ocean Wave Statistics, (1967, [19]), presents a statistical breakdown showing the number of observàtions in various wave heights versus period categories. These statistics represent actual observations from ships. The observations are from an area somewhat larger than the area choscn. for sitc analyais, but the conditions over the area are represented as being homogeneous. Figure VI-l provides a graph of the relative frequency of various wave heights for the Mombasa site.

'l'able VI-4 summarizes mean wave data for the Mombasa site from Climatic Summariès For Major Indian Ocean Ports $(1974, \underline{35}])$. 

TABLE VI-3: STATISTICAL BREAKDOWN SHOWING NUMBER OF SHIPS OBSERVATIONS
OFF MOMBASA REPORTING VARIOUS HEIGHT/PERIOD

COMBINATIONS (ALL SEASONS). From Ocean Wave Statistics (1967), [19].

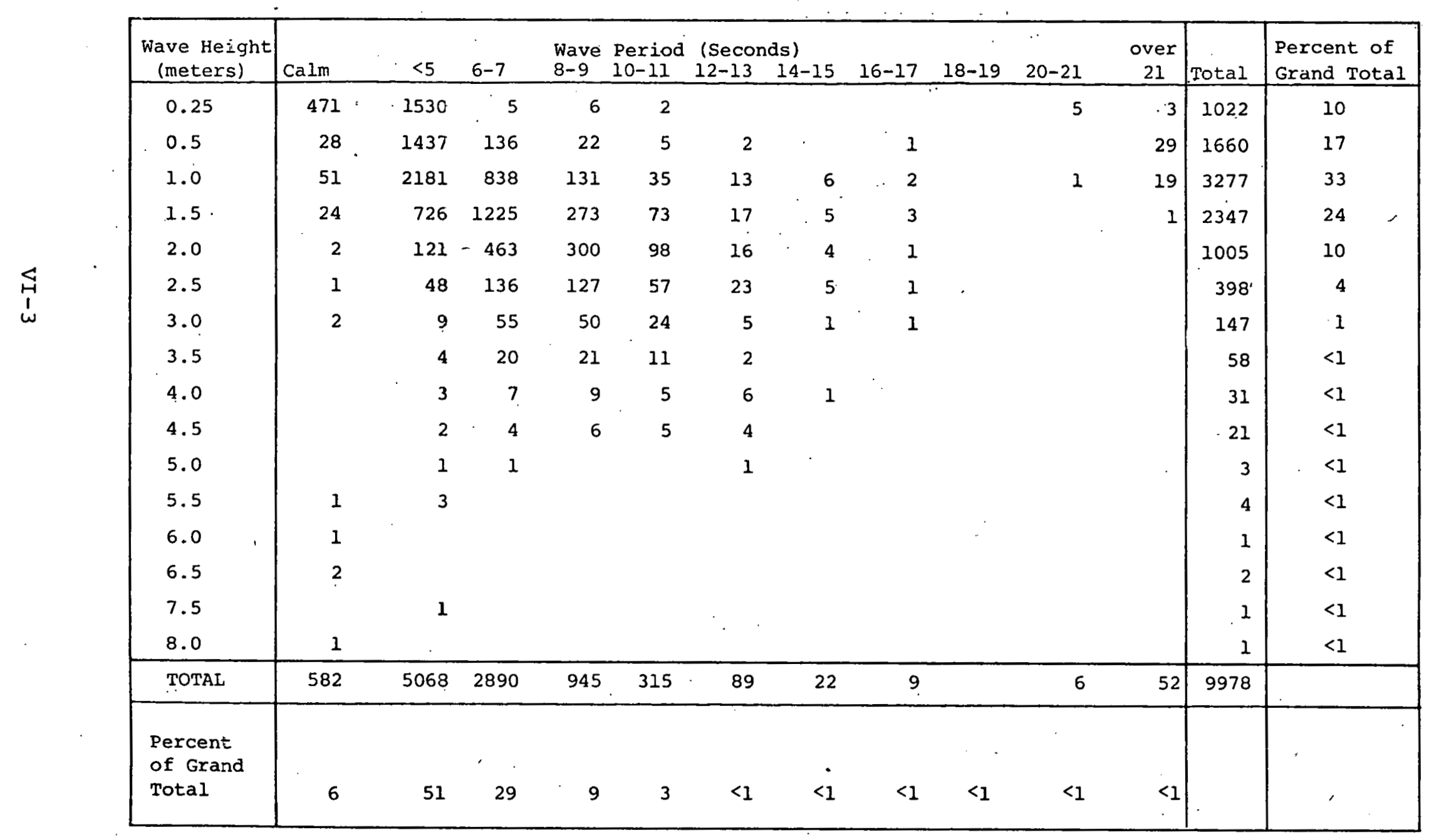




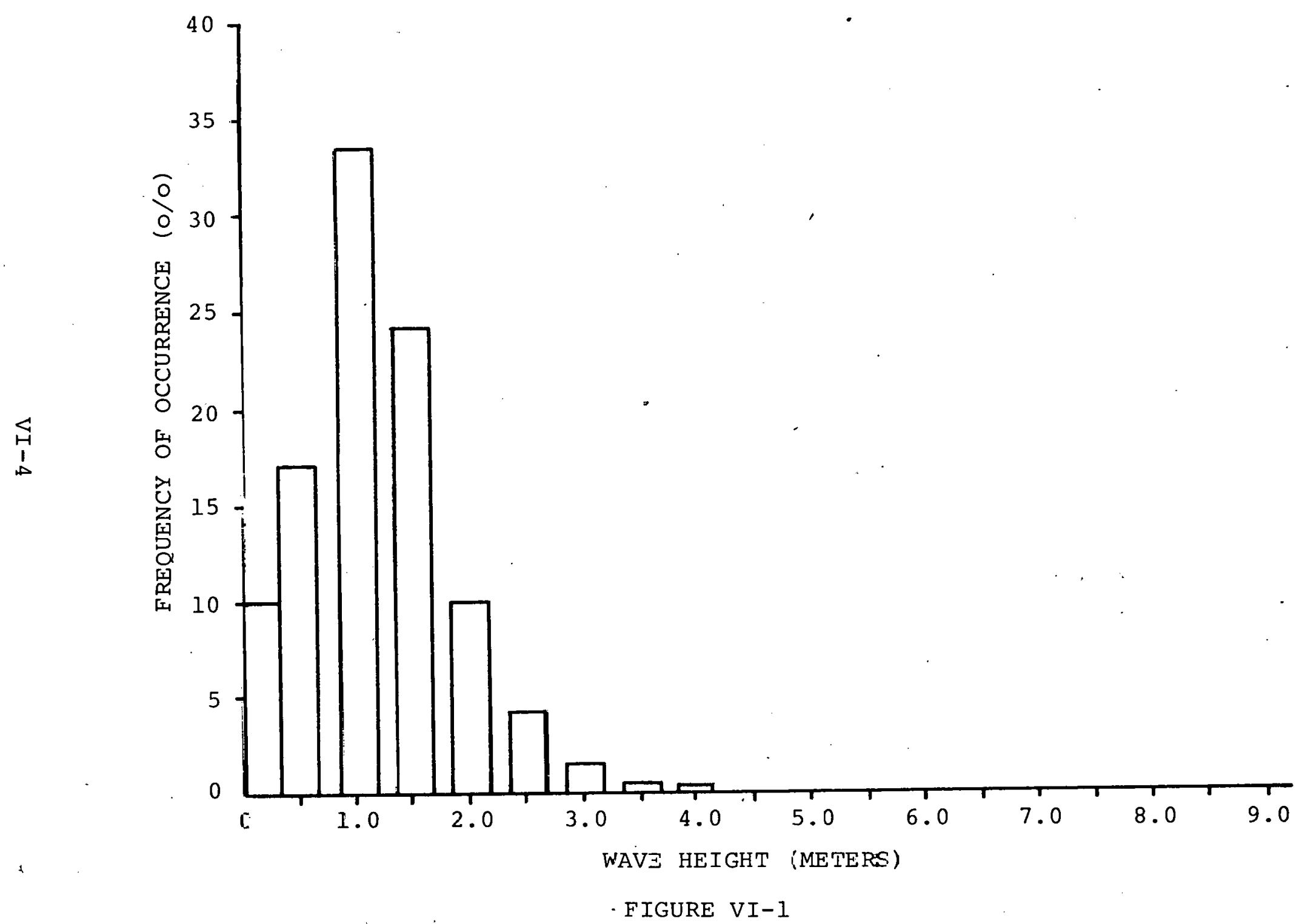

WAVE HEIGHT FREQUENCIES (MOMBFSA) 
TABLE VI-4: MONTHLY MEAN WAVE HEIGHTS IN METERS.

\begin{tabular}{|c|c|c|c|c|c|c|}
\hline \multirow{4}{*}{$\begin{array}{l}\text { Northern } \\
\text { Section }\end{array}$} & JAN & $\underline{F E B}$ & MAR & $\underline{\mathrm{APR}}$ & $\underline{M A Y}$ & JUN \\
\hline & $1.2-1.5$ & $1.2-1.5$ & $0.6-0.9$ & $0.6-0.9$ & $1.2-1.5$ & $1.8-2.1$ \\
\hline & JUL & AUG & SEP & OCT & NOV & DEC \\
\hline & $1.8-2.1$ & $1.2-1.5$ & $1.2-1.5$ & $0.6-1.5$ & $0.6-0.9$ & $0.6-1.5$ \\
\hline \multirow{4}{*}{$\begin{array}{l}\text { Southern } \\
\text { Section }\end{array}$} & JAN & FEB & MAR & APR & MAY & JUN \\
\hline & $1.2-1.5$ & $0.6-1.5$ & $0.6-0.9$ & $0.6-0.9$ & $1.2-1.5$ & $1.2-2.1$ \\
\hline & JUL & $\underline{\Delta U G}$ & SEI & OCT & NOV & DEC \\
\hline & $1.2-2.1$ & $1.2-1.5$ & $1.2-1.5$ & $1.2-1.5$ & $0.6-0.9$ & $0.6-1.5$ \\
\hline
\end{tabular}


VII. CURRENTS

The circulation pattern of the area responds to seasonal shifts in the monsoons. The surface currents during the summer and winter seasons have definite characteristics. During the Northeast Monsoon, water north of the equator is carried westward by the Northeast Monsoon current. This water flows southward along Somalia; west of Madagascar the south equatorial current branches into the northward-flowing East African Coast Current. This East African Coast Current converges with the southward-flowing somalia current. From the convergence zone, the eastward-flowing Equatorial countercurrent begins" in November-December.

During the Southwest Monsoon, most of the South Equa- . torial Current proceeds into the Somali Current moving northward along Kenya and Somalia.' Unlike the weak southwardflowing Somali Current during the Northwest Monsoon, this Somali Current is quite strong. North of the equator, it turns eastward into the Southwest Monsoon Current. The reversal to the Southwest Monsoon region begins in AprilMay, usually in April south of the equator.

Iike mean wind speeds during the Northeast Monsoon, currents during this period are generally weak and variable. Only in the southernmost part of the section off the city of Mombasa are lie surface currents of strength, generally about $50 \mathrm{~cm} / \mathrm{sec}$, but occasionally near $100 \mathrm{~cm} / \mathrm{sec}$. The currents due east of the city of Mombasa at a surface 
station where the bottom is 1000 meters deep show very consistent flow toward the northeast at all depths. This station is in the East African Coast Current. The surface flow is around $50 \mathrm{~cm} / \mathrm{sec}$ with little variability in speed and direction between January and July. The speeds of the currents decrease further north along the coast. Off Somalia, surface current speeds are generally closer to $10 \mathrm{~cm} / \mathrm{sec}$, between January and June. During the summer conditions, surface current speeds of $50 \mathrm{~cm} / \mathrm{sec}$ or greater occur in the entire region. This current information is from Duing and Schott, 1978, [15].

The rare hurricane which occurs in this region will affect surface currents. Leipper, 1967 [21] showed that a current had developed in the area transversed by Hurricane Hilda. Analysis of temperature - depth data showed a current of approximately $50 \mathrm{~cm} / \mathrm{sec}$. A theoretical study by O'Brien and Reid, 1967 [27], states that hurricanes will cause currents with a speed of approximately one meter per second.

The passage of hurricanes can induce upwelling of subsurface water for a temporary period. This upwelling will cause anomalous vertical current shears. Strong vertical and horizontal current shears are also observed in association with major current systems. This site is in the source region of the Somali. current, so there may be strong current shears off Mombasa. 
1. Adamec, D., and O'Brien, J.J., November 1978, "The Seasonal Upwelling in the Gulf of Guinea Due to Remote Forcing", J. Phys. Oceanogr., 8, No. 6, 1050-1060.

2. Atwood, D.K., et al., 1976, Ocean Thermal Energy Conversion, Resource Assessment and Environment Impact for Proposed Puerto Rico Site, University of Puerto Rico.

3. Avery, W.H., et al., 1976, Maritime and Construction Aspects of Ocean Thermal Energy Conversion (OTEC) Plant Ships, The John Hopkins University Applied Physics Laboratory, Laurel, Maryland.

4. Bathen, K.H., et al., 1977, "Consolidated Oceanographic and Meteorological Data for Four North Pacific OTEC Sites", University of Hawaii.

5. Bigelow, H.B., and W.T. Emundson, 1947, Wind waves at Sea, U.S. Hydrographic office, Washington, D.C.

6. Colburn, J.G., 1974, "The Thermal Structure of the Indian Ocean", International Indian Ocean Expedition Oceanographic Monograph No., 2; The University Press of Hawaii; Honolulu, Hawaii.

7. Crutcher, H.L. and R.G. Quayle, 1974, "Mariners Worldwide Climatic Guide to Tropical storms at Sea", NAVAIR 501C-61, Naval Weather Service; Asheville, N.C.

8. Defense Mapping Agency Hydrographic/Topographic Center, 1978, Pilot Chart of the North Pacific, DMA Stock Numbers PILOT 557801, 557804,557810, Washington, D.C.

9. Defense Mapping Agency Hydrographic/Topographic Center, 1978, Pilot Chart of the North Atlantic Ocean, DMA Stock Numbers PILOT 167801, 167804, 167807, 167810, Washington, D.C.

10. Defense Mapping Agency Hydrographic Center, South Pacific Ocean, Sheet IV, Pub. N.O. 623, revised 1974, Washington, D.C.

11. Defense Mapping Agency Hydrographic Center, Indian Ocean, Northern Part, Pub No. 721, 1974, Washington, D.C.

12. Defense Mapping Agency Hydrographic Center, Gulf of Mexico and Carribean Sea, Pub. No, 410, revised 1973, Washington, D.C. 
13. Defense Mapping Agency Hydrographic Center, North Atlantic Ocean, Southeastern Sheet, Publication N.0. 125, 4th ed., 1974, Washington, D.C.

14. Düing, W., 1970; "The Monsoon Regime of the Currents in the Indian Ocean", International Indian Ocean Expedition Oceanographic Monograph No. 1, Hawaii Institute of Geophysics Contribution No. 331, the University of Hawaii Press; Honolulu, Hawaii.

15. Düing, W. and F. Schott; March 1978, "Measurements in the Source Region of the Somali Current during the Monsoon Reversal", J. Phys. Oceanogr., 8, 278-289.

16. Emilsson, J., 1970, "On the Upper Layer Circulation in the Cayman Sea", from the symposium on the Investigations and Resources of the Caribbean Sea and Adjacent Regions, UNESCO, Paris France.

17. Fleel Numerlcal Weather Central (FNWC), FNWC and National Oceanographic Data Center digitized reports.

18. Gentry, R.C., 1970, "Hurricanes, One of the Major Features of Air-Sea Interaction in the Caribbean Sea", from the Symposium on the Investigation and Resources of the Caribbean Sea and Adjacent Regions, UNESCO, Paris, France.

19. Hogben, N., and F. Lumb, 1967, Ocean Wave Statistics, National Physical Laboratory, Ministry of Technology, London. Her Majesty's Stationery Office.

20. Johnson, A. and S. Denwick, 1978; "Data Report Buoy Ubservations During Hurricanes Anita and Babe, AugustSeptember, 1977", NOAA Data Buoy Office, National Space Technology Laboratories, NSTL Station; Mississippi.

21. Leipper, D.F., 1967, "Obscrved Ocean Cunditions and hurricane Hilda, 1964. J. Atmos Sci., 24, p 182-196.

22. MCFadden J.D., "Airborne Investiqations of the Effects of Hurricanes on the Thermal Structure of the surface Layer of the Ocean", from the Symposium on Investigation and Resources of the Caribbean Sea and Adjacent Regions, UNESCO, Pal is France.

23. National Oceanographic Data Center, The Variability of Water Masses in the Indian Ocean, Fublication G-11, Washington, D.C. 
24. National Oceanographic Data Center, Data File BTG75A, Washington, D.C., 1977 .

25. National Oceanographic Data Center, Data Files SD40A5/0A6 from SD76A (1/30/76) 3509 STA: Washington, D.C., 1977.

26. National Science Foundation, 1972, Meteorological Atlas of the International. Indian Ocean Expedition, Volume 7, Washington, D.C.

27. O'Brien, J.J., and R.O. Reid, 1967, "The Non-Linear Response of a Two Layer Baroclinic Ocean to a Stationery Axially Symetric Hurricane", J. Atmos. Sci., 24, p 197215 .

28. Ramage, C.D., 1972, "Indian Ocean Surface Meteorology", International Indian Ocean Expedition, Collective Reprints VIII, Contribution No. 624, pgs 407-540; Paris, France.

29. Sirvastava, P.S., P.K. Vyayarayou and M.X. Joseph, 1972

"Monthly Wave Characteristics of the Bay of Bengal", International Indian Ocean Expedition, Collective Reprints VIII, Contribution No. 625, Paris, France.

30. The Enclyopedia of Oceanography, 1966, Reinhold Publishing Corp., New York, New York.

31. U.S. Department of Agriculture, Weather Bureau, 1938, "Atlas of Climatic Charts of the Oceans", Washington, D.C.

32. U.S. Department of Commerce, NOAA, 1971, Eastropac Atlas, Volume 3, Washington, D.C.

33. U.S. Navy, 1975, Marine Climatic Atlas of the World, Volume III, Indian Ocean, Washington, D.C.

34. U.S. Navy Hydrographic office, 1964, Atlas of Sea and Swell Charts, Northeastern Pacific Ocean, Publication No. 799D, revised 1976, Washington, D.C.

35. U.S. Naval Weather Service, September 1974, Climatic Summaries For Major Indian Ocean Ports and Waters, NAVAIR 50-1C-63, Asheville, N.C.

36. U.S. Naval Weather Service, November 1973, Climatic Summaries For Major Seventh Fleet Ports and Waters, NAVAIR 50-1C-62, Ashevilie, N.C.

37. U.S. Naval Oceanographic Office, 1963, Oceanographic Atlas of the North Atlantic Ocean, Washington, D.C. 
38. U.S. Naval Oceanographic Office; Bathymetric Atlas of' the Northeastern Pacific Ocean, Pub No. 1303-S, No. $0902 \mathrm{~N}, 0903 \mathrm{~N}, 1002 \mathrm{~N}, 1003 \mathrm{~N}$, Washington, D.C.

39. U.S. Naval Oceanographic Office, Indian Ocean, AfricaEast Coast Publication No. 724, lst ed., 1974, Washington, D.C.

40. U.S. Naval Oceanographic Office, 1971, Bathymetric Atlas of the Northeastern Pacific Ocean, H.O. Pub No. $1303-\mathrm{S}$, N.O. $1104 \mathrm{~N}, 1105 \mathrm{~N}, 1204 \mathrm{~N}, 1204 \mathrm{~N}$, Washington, D.C.

41. U.S. Naval Oceanographic Office, 1971, Bathymetric Atlas of the Northeastern Pacific Ocean, H.O. Pub N.O. $1301-\mathrm{S}, 2403 \mathrm{~N}, 2040 \mathrm{~N}$, Washington, D.C.

42. U.S. Naval Oceanographic Office, 1970, Bathymetric Atlas of the Northwestern Parifis Dsean, H:O. Fub. 1201-S, N.O. 2203N, Washington, D.C.

43. Wolff, P.M. and W.E. Hubert, 1976, "Ocean Thermal Energy Conversion: Resource, Ecological and Environmental Studies", Contract No. NSF-C1020, Ocean Data Systems, Inc., Monterey, California.

44. Wolff, P.M., et al, 1977, "OTEC Resource Report for Hawaii", Contract EG-77-C-01-4028, Ocean Data Systems, Inc., Monterey, California.

45. Wyrtki, K., 1972, "The Upwelling in the Region Between Java and Australia during the South-East Monsoon, Collected reprints of the International Indian Ocean Expedition, Volume I, Contribution No. 15, pp 151-161.

46. Wyrtki, K., 1972, "Geopotential Topographics and Associated Circulation in the South Eastern Indian Ocean", Collerter reprints of tlit International Indian Ocean Expedition, Volume $I$, contribution No. 14 .

47. Wyrtki, K., 1971, Oceanographic Atlas of the International Ocean Expedition, National Science Foundation, NSF-IOE1, Washington, D.C. 